\title{
Component Importance Measure Computation Method Based Fuzzy Integral with Its Application
}

\author{
Shuai Lin, ${ }^{1,2}$ Yanhui Wang, ${ }^{1,3}$ Limin Jia, ${ }^{1,3}$ and Yang $\mathrm{Li}^{1,2}$ \\ ${ }^{1}$ State Key Laboratory of Rail Traffic Control and Safety, Beijing Jiaotong University, Beijing 100044, China \\ ${ }^{2}$ School of Traffic and Transportation, Beijing Jiaotong University, Beijing 100044, China \\ ${ }^{3}$ Beijing Research Center of Urban Traffic Information Sensing and Service Technologies, Beijing Jiaotong University, \\ Beijing 100044, China \\ Correspondence should be addressed to Shuai Lin; linshuai2016gmu@gmail.com
}

Received 13 February 2017; Accepted 22 June 2017; Published 17 August 2017

Academic Editor: Manuel De la Sen

Copyright (C) 2017 Shuai Lin et al. This is an open access article distributed under the Creative Commons Attribution License, which permits unrestricted use, distribution, and reproduction in any medium, provided the original work is properly cited.

In view of the negative impact of component importance measures based on system reliability theory and centrality measures based on complex networks theory, there is an attempt to provide improved centrality measures (ICMs) construction method with fuzzy integral for measuring the importance of components in electromechanical systems in this paper. ICMs are the meaningful extension of centrality measures and component importance measures, which consider influences on function and topology between components to increase importance measures usefulness. Our work makes two important contributions. First, we propose a novel integration method of component importance measures to define ICMs based on Choquet integral. Second, a meaningful fuzzy integral is first brought into the construction comprehensive measure by fusion multi-ICMs and then identification of important components which could give consideration to the function of components and topological structure of the whole system. In addition, the construction method of ICMs and comprehensive measure by integration multi-CIMs based on fuzzy integral are illustrated with a holistic topological network of bogie system that consists of 35 components.

\section{Introduction}

Recent decades have witnessed not only the rapid development on the highly integrated system of electromechanical systems, but also the significant progress on the system function [1]. Complex electromechanical systems, such as high-speed train, aircraft, and other large equipment, are composed of components with specific functions, physical and chemical connections, and behaviors, and coupled relationship through mechanic, electric, and information relationship. Due to the complexity of topological structure and functional relationship within electromechanical systems, one of the component's failures may lead to another component's failure, which is called fault propagation. The fault propagation of complex electromechanical systems can enlarge the negative impact due to one of components failures. In these situations, it is becoming increasingly important to take functional and topological characteristics into account when assessing the importance of components and concentrating the resources on the small subset of components that are most important to the system.

1.1. Previous Work. In system reliability theory, importance measures are used as effective tools to evaluate the relative importance of components and identify system weaknesses [2]. Component importance measures (CIMs) are component related indices that allow security practitioners to identify how a components fault affects the overall behavior or performance of the whole technological system and are used to evaluate the relative importance of a component. The typical CIMs include but are not limited to Birnbaum importance measure [3], Fussell-Vesely (FV) importance measure [4], and criticality importance measure [5]. Detailed descriptions and mathematical expressions for importance measures can be found in Ramirez-Marquez [6]. Using the CIMs, security practitioners can estimate or prioritize 
components in order of their importance value with regard to system reliability and concentrate maintenance resources on the most important components.

Recent advances indicate that electromechanical systems can be virtually represented as networks, where the components of technological products are easily depicted by the nodes of complex networks and the connections between linkage components are naturally depicted by the links of complex networks [7-9]. More recently, various centrality measures (CMs) have been presented to quantify the importance of an individual in a complex network, including degree centrality (DC) [10], betweenness centrality (BC) [11], and eigenvector centrality (EC) [12]. The issue of centrality has attracted the attention of physicists, who have been extending its applications to the realm of technological networks. For example, Dan et al. [13] considered that system networked reflected the organization structure and enhanced efficiency and capability of system development and production, and, in $\mathrm{Li}$ et al. [14] view, these physical connections between components determine the function and structure complexity of technological products. Based on CMs of complex network, Jiang et al. [15] introduced the loads and vulnerability coefficient of nodes to study the inherent vulnerability of components and $\mathrm{Xu}$ et al. [16] developed a comprehensive vulnerability index to find the vulnerable structure of complex system with a network model. Meanwhile, Zong et al. [17] regarded the node betweenness and node agglomeration as the indices to evaluate the importance of the components based on the maintenance relationship network.

However, all these researches focused on only one measure, such as component importance measure and one centrality measure, and every measure has its own disadvantage and limitation. In recent years, researchers study a multiattribute ranking problem to evaluate the component importance comprehensively from more than one perspective, which would be a special case of multicriteria decisionmaking (MCDM). MCDM refers to making decision for alternatives in the presence of multiple and conflicting criteria [18] and has many developments and applications, such as extensions of TOPSIS [19, 20], Analytic Hierarchy Process [21], K-shell decomposition [22], and entropy theory [23]. Detailed descriptions and mathematical expressions for multicriteria decision-making approaches can be found in Govindan et al. [24].

1.2. Problem Description. Although the above component importance measures or centrality measures have been widely applied in identifying influential components, there are some limitations and disadvantages. CIMs are built on the assumption of the independence of components and none of them has taken the impact of topological structure between components into account. CMs mentioned above focus only on the components propagation behavior of complex network and are limited to the point of the reliability analysis [25]. For these reasons, it is extremely important to research on the negative impact of these restrictions and proactively overcome them by complementation with elaborate reliability contexts on identifying influential components of electromechanical system. That is to say that CIMs or CMs cannot be applied to complex electromechanical systems that contain multiple components.

If only one measure is adopted, then the rankings of identifying influential components may be different by using a different measure. In some cases, using different centrality measures may provide different results, even conflicting results [26]. MCDM has been proposed to address this problem. However, the inherent limitations and disadvantages of CIMs or CMs cannot be eliminated through integration multimeasures. Moreover, among numerous MCDM methods developed to solve real-world decision problems, fuzzy integral continues to work satisfactorily across different application areas. The weights in most developments and applications of MCDM are determined in advance, such as TOPSIS and AHP, which possess definite subjectivity. Fuzzy integral makes full use of attribute information, provides a cardinal ranking of alternatives, reduces subjective influences, and does not require attribute preferences to be independent. As a well-known classical MCDM method, fuzzy integral has received much interest from researchers and practitioners.

In this paper, we try to introduce fuzzy integral theory to explore how to identify influential components. Our work makes two important contributions. First, we integrate component importance measures and centrality measures with Choquet integral to define a new kind of improved centrality measures. Second, a novel index, comprehensive measure, of a meaningful fuzzy integral-based is brought into identification of important components for which it could give consideration to function of components and topological structure of the whole system.

The rest of the paper is structured as follows. Section 2 provides background information about the holistic topological network and fuzzy integral theory. In Section 3, the improved centrality measures are detailed. The following is presented: how to improve centrality measures, by using Choquet integral to express functional and topological properties which are related, measuring the importance of components in electromechanical systems. In Section 4, we embed the fuzzy integral into the process of construction comprehensive measure and identification of critical components by fusing multi-improved centrality measures. Section 5 presents a case study which constructs improved centrality measures of bogie system and discusses the advantages of fusion improved centrality measures in identifying critical components based on fuzzy integral.

\section{Methodological Background}

2.1. The Holistic Topological Network. Currently, complex networks are being studied in many fields of science, such as social sciences, computer sciences, physics, biology, and economics. The majority of systems in reality can be undoubtedly described by models of complex networks. For example, Internet is a complex network composed of web sites [27, 28]. The brain is a complex network of neurons [29]. An organization is a complex network of people [30]. 


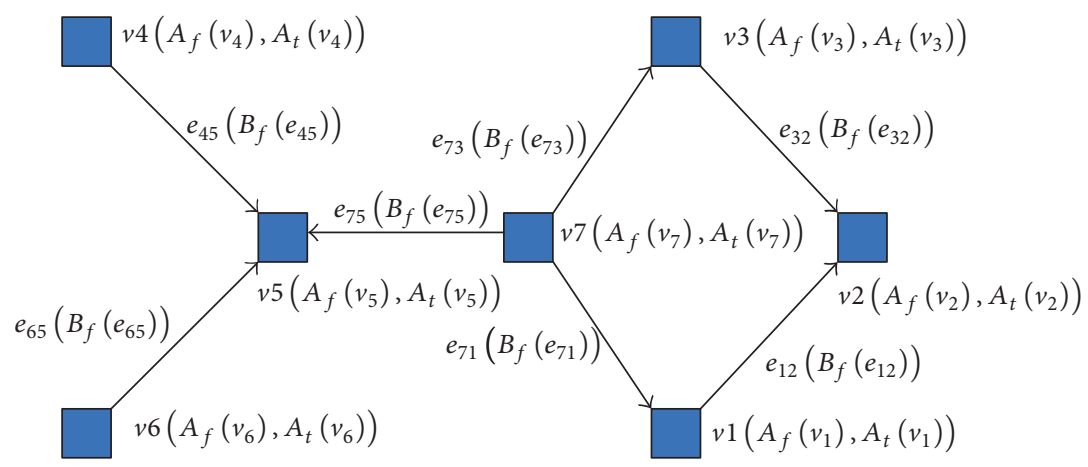

FIgURE 1: The holistic topological network.

As mentioned above, electromechanical systems are characterized by large scale, complex structure, nonlinear behavior, various working states, high coupling components, random operation environment, and so forth, which are not easy to be modeled directly to analyze global behaviors. Recently, lots of attempts have been made to model network in engineering, more specifically, electromechanical systems. It can be a complex network, in which the components are depicted by nodes and the physical connections between linkage components are depicted by links between the corresponding nodes. We refer to this representation as a topological structure and a formal model is presented by the following definitions.

We define HTN $=\langle V, E, f(V), f(E)\rangle$ as a holistic topological network

$$
\begin{aligned}
& \mathrm{HTN}=\langle V, E, f(V), f(E)\rangle \\
& \qquad v_{i} \in V, e_{i j} \in E, f\left(v_{i}\right)=\left\{A_{f}\left(v_{i}\right), A_{t}\left(v_{i}\right)\right\} \subset f(V), f\left(e_{i j}\right)=\left\{B_{f}\left(e_{i j}\right)\right\} \subset f(E), i=1, \ldots, n ; j \leq n,
\end{aligned}
$$

where $V=\left\{v_{1}, v_{2}, \ldots, v_{n}\right\}$ is a set of nodes and each node represents a component. $n$ is the number of nodes. For example, the number of nodes is 7 for a system in Figure 1. $E=\left\{e_{i j} \mid i=1, \ldots, n, j \in n\right\}$ is a set of links and $e_{i j}$ represents physical connection between nodes $v_{i}$ and $v_{j}$. Depending on the nature or type of the topological property, this property may be reflexive in that $e_{i j} \neq e_{j i} . f(V)$ is the properties of the set of nodes, $A_{f}\left(v_{i}\right)$ is the functional properties of $v_{i}$, and $A_{t}\left(v_{i}\right)$ is the topological properties of $v_{i} . f(E)$ is the properties of the set of edges and $B_{f}\left(e_{i j}\right)$ is the functional properties of edge $e_{i j}$.

For different systems, the properties of nodes or edges may be also different. For instant, assuming that Figure 1 is the partial bogie system of CRHX, the properties of nodes and edges in the holistic topological network are as follows.

(1) $A_{f}\left(v_{i}\right)=\left\{\mathrm{LT}_{i}, \mathrm{MTBF}_{i}, \lambda_{i}\right\}$. $\mathrm{LT}_{i}$ is the service life of node $v_{i}$, which is its expected lifetime or the acceptable period of use in service. It is the time that any manufactured item can be expected to be "serviceable" or supported by its manufacturer. We can obtain this parameter from its manufacturer.

$\lambda_{i}$ is failure rate of node $v_{i}$ and is defined as

$$
\lambda_{i}(t)=\lim _{\Delta t \rightarrow 0} \frac{R_{i}(t)-R_{i}(t+\Delta t)}{\Delta t \cdot R_{i}(t)},
$$

where $R_{i}(t)$ is the probability of no failure before time $t$ for node $v_{i} ; \Delta t$ is the over-a-time-interval from failure time $t$ to the next failure time. In this paper, failure rate $\lambda_{i}$ depending on failure data is computed.

$\mathrm{MTBF}_{i}$ is mean time between failures of node $v_{i}$ and the formula is

$$
\operatorname{MTBF}_{i}=\frac{\sum\left(f_{i}^{d}-f_{i}^{u}\right)}{N_{i}^{f}},
$$

where $f_{i}^{d}$ is start of downtime for node $v_{i}, f_{i}^{u}$ is start of uptime for node $v_{i}$, and $N_{i}^{f}$ is the number of failures.

(2) $A_{t}\left(v_{i}\right)=\left\{\mathrm{BC}_{i}, \mathrm{CC}_{i}, \mathrm{DC}_{i}, \mathrm{EC}_{i}\right\} . \mathrm{CC}_{i}$ is closeness centrality [31] of node $v_{i}$ :

$$
\mathrm{CC}_{i}=\frac{1}{\sum_{j}^{n} d_{i j}},
$$

where $d_{i j}$ denotes the distance between node $v_{i}$ and node $v_{j}$; $n$ is the number of nodes.

$\mathrm{BC}_{i}$ is betweenness centrality [31] of node $v_{i}$ :

$$
\mathrm{BC}_{i}=\sum_{j, k \neq i} \frac{g_{j k}(i)}{g_{j k}},
$$

where $g_{j k}$ is the number of binary shortest paths between node $v_{j}$ and node $v_{k}$ and $g_{j k}(i)$ is the number of those paths that go through node $v_{i}$. 
$\mathrm{DC}_{i}$ is degree centrality [32] of node $v_{i}$ :

$$
\mathrm{DC}_{i}=\sum_{j}^{N} x_{i j}
$$

where $i$ is the focal node $v_{i} ; j$ represents all other nodes; $N$ is the total number of nodes; $x_{i j}$ represents the connection between node $v_{i}$ and node $v_{j}$. The value of $x_{i j}$ is defined as 1 if node $v_{i}$ is connected to node $v_{j}$ and 0 otherwise.

$\mathrm{EC}_{i}$ is eigenvector centrality [12] of node $v_{i}$. Let $A$ be a $n \times n$ similarity matrix. The eigenvector centrality $\mathrm{EC}_{i}$ of node $v_{i}$ is defined as the ith entry in the normalized eigenvector belonging to the largest eigenvalue of $A$. $\lambda$ is the largest eigenvalue of $A$ and $n$ is the number of vertices:

$$
\begin{aligned}
A \cdot \mathrm{EC} & =\lambda \cdot \mathrm{EC} \\
\mathrm{EC}_{i} & =\mu \sum_{j=1}^{n} a_{i j} \mathrm{EC}_{j}, \quad i=1, \ldots, n
\end{aligned}
$$

with proportionality factor $\mu=1 / \lambda$ so that $\mathrm{EC}_{i}$ is proportional to the sum of similarity scores of all nodes connected to it.

(3) $B_{f}\left(e_{i j}\right)=\left\{p_{i j}, \mathrm{st}_{i j}, \lambda_{i j}\right\} \cdot p_{i j}$ is fault propagation probability of edge $e_{i j}$, given by

$$
p_{i j}=\frac{l\left(e_{i j}\right)}{\sum_{i \neq j} l\left(e_{i j}\right)},
$$

where $l\left(e_{i j}\right)$ is the number of shortest paths crossing a given edge $e_{i j}$. Gao et al. [33] introduced the concept and computational method of fault propagation probability in detail. 35]

st $_{i j}$ is connection strength of edge $e_{i j}$ and is given by [34,

$$
\mathrm{st}_{i j}=\beta \frac{s\left(v_{i} \mid v_{j}\right)}{s\left(v_{i}\right)},
$$

where $s\left(v_{i}\right)$ is the number of times that operation states change in the statistical time; $s\left(v_{i} \mid v_{j}\right)$ indicates the number of times that $v_{i}$ operation states change arising from $v_{i}$ in the statistical time. The operation states of $v_{i}$ contain the failure mode and normal operation mode of the corresponding component. $\beta$ is an empirical contact duration of the type of functional dependencies between components $v_{i}$ and $v_{j}$.

$\lambda_{i j}$ is failure rate of edge $e_{i j}$. The calculation method is the same as the failure rate of node in (2).

In Figure 1, the edge describes the physical connection between linkage components, and the arrow of the edges expresses the failure interaction of nodes. Liu and An [22] further introduced how to determine the direction of edges which describe the coupling relation of the failure interactions.

2.2. Fuzzy Integral Theory. About thirty years ago the concept of fuzzy integral was proposed in Japan by Sugeno $[36,37]$, which in the discrete case is merely a kind of distorted mean. Although this was followed by a rather mathematically oriented research, far from application concerns, some Japanese researchers, including Sugeno himself, thought that fuzzy integrals could be applied to multicriteria evaluation: since 1985, papers have been published on supplier evaluation and improvement [38], analysis of policy decision of sustainable energy strategies [39], fusion of extreme learning machine [40], analysis of human reliability [41], and so forth. The distinguishing feature of fuzzy integral is that it is able to represent a certain kind of interaction between criteria, ranging from redundancy (negative interaction) to synergy (positive interaction). To our knowledge, there is almost no well-established method to deal with interacting criteria, and usually people tend to avoid the problem by constructing independent (or supposed to be so) criteria. This innovative feature was without any doubt the reason of its success in various fields of application [42]. A wide variety of forms have been presented for fuzzy integral, such as Choquet integral [43] and Sugeno integral.

Let $X=\left\{x_{1}, x_{2}, \ldots, x_{n}\right\}$ be a nonnull and finite set of attributes or influence factors and let $P(X)$ denote the power set of $X$.

2.2.1. Discrete Choquet Integral. Let $g_{\lambda}$ be a $\lambda$ fuzzy measure in $P(X)$. The discrete Choquet integral of an element $f: X \rightarrow$ $I R^{+}$with respect to $g_{\lambda}$ is defined by

$$
\text { (c) } \int f d g_{\lambda}=\sum_{i=1}^{n}\left(f\left(x_{(i)}\right)-f\left(x_{(i-1)}\right)\right) g_{\lambda}\left(A_{(i)}\right),
$$

where $(i)$ indicates a permutation on $X$ such that $f\left(x_{(1)}\right) \leq$ $\cdots \leq f\left(x_{(n)}\right)$. Also $A_{(i)}=\left(x_{(i)}, \ldots, x_{(n)}\right)$ is an attribute set, and $f\left(x_{(0)}\right)=0$. Discrete Choquet integral is the extension of the weighted average operator, which considers the relationships among attributes or influence factors.

2.2.2. Discrete Sugeno Integral. Let $\mu$ be a fuzzy measure on $X$ and $f: X \rightarrow[0,1]$ be a function. The discrete Sugeno integral of $f$ with respect to $\mu$ is

$$
(s) \int f d \mu=\bigvee_{i=1}^{n}\left(f\left(x_{(i)}\right) \wedge \mu\left(A_{(i)}\right)\right),
$$

where $0 \leq f\left(x_{(i)}\right) \leq f\left(x_{(2)}\right) \leq \cdots \leq f\left(x_{(n)}\right) \leq 1, A_{(i)}=\left(x_{(i)}\right.$, $\left.x_{(i+1)}, \ldots, x_{(n)}\right)$ is an attribute set and $f\left(x_{(0)}\right)=0$.

\section{Improved Centrality Measures}

In this section, the novel importance measures, that is, improved centrality measures (ICMs), are first proposed. It is a series of special importance measures to find the influential components that are really crucial for the normal operation of electromechanical systems. When some components lose or weaken their functions due to a certain mode of failures, there will be a degradation of the holistic system performance. Based on centrality measures, the originality and novelty of proposed ICMs is that they evaluate the importance of a 


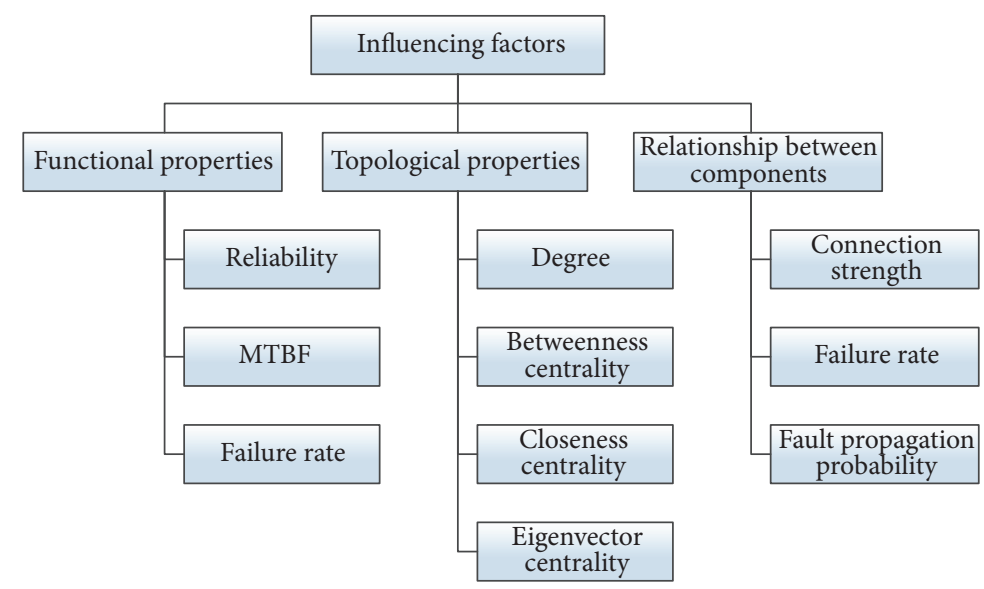

FIGURE 2: Influencing factors of the individual components.

component by taking into account the functional and topological properties in the holistic system. In essence, they are comprehensive indicators in the system, which are proposed for the assessment of the most important component.

3.1. Influencing Factors. In order to measure the importance of components, influential factors that are capable of representing the desired function of the individual components, as well as their structure, are required. Some quantitative information is introduced into the system to improve the accuracy of the important measure of the components. For example, functional properties reflect the ability of the system to perform its intended function, and topological properties describe the stability of the inherent structure of the system.

Through the project (The National High Technology Research and Development Program of China (863 Program) number 2012AA112001) of cooperation with CRRC Corporation Limited, we have discussed with engineers and maintenance personnel in detail and then obtained the influential factors of bogie system as shown in Figure 2, which include the functional properties, which are used to depict the system performance, and the topological properties, which are used to describe the influence on system structure.

3.2. Improved Centrality Measures. A large number of centrality measures have been proposed to identify influential nodes within a complex network. Examples are DC, CC, BC, and EC. However, it cannot be ignored that while most of these centrality measures have been widely used in Network Reliability Analysis, challenges still remain in regard to the following: none of them has taken into account the impact of functional influences of nodes and edges in the holistic topological network.

Improved centrality measures are a series of importance measures which are extended from centrality measures. In a sense, ICMs can serve as better importance measures of components, as it synthesizes the components functional properties, such as usage reliability, failure rate, and connection strength, meanwhile, by taking systems structure into account, such as CC, BC, DC, and EC.
The following is an efficient and universal construction method to calculate ICMs.

Let us consider $A_{f}\left(v_{i}\right)$ in (1), which consists of functional properties of nodes, $A_{t}\left(v_{i}\right)$, which consists of topological properties of nodes, and $B_{f}\left(e_{i j}\right)$, which consists of functional properties of edges.

Based on the holistic topological network, functional properties of nodes and edges are integrated into the definition of centrality measures, and then ICMs are constructed, defined as follows:

$$
\begin{array}{r}
\mathrm{ICM}_{s}(i)=(c) \int\left(x_{s}(i),(c) \int A_{f}(i) d \mu_{A_{f}},(c)\right. \\
\left.\cdot \int B_{f}\left(e_{i j}\right) d \mu_{B_{f}}\right) d \mu_{\mathrm{ICM}} \\
x_{s}(i) \in A_{t}(i),
\end{array}
$$

where $\operatorname{ICM}_{s}(i)$ is the improved centrality measure of node $v_{i}$. $\mu$ is the weight of influencing factors.

According to (12), the first thing that we need is to calculate weight $\mu$. Here, we use $g$ fuzzy measure and $k$ additive fuzzy measure to compute weight $\mu$ based on Shapley values [44] and Marichal entropy theory [45]. The maximum Marichal entropy as the objective function constructs optimization model as shown in (13) based on fuzzy theory. And then, calculating (13), we can get the parameter $\mu$.

$$
\begin{array}{ll}
\max _{\lambda, \mu} & H_{M}(\mu)=\sum_{i=1}^{n} \sum_{S \subseteq A-a_{i}} \gamma_{s}[n] \cdot h\left[\mu\left(S \cup a_{i}\right)-\mu(S)\right] \\
\text { s.t. } & I_{i} \\
& =\sum_{k=0}^{n-1} \frac{(n-k-1) ! k !}{n !} \sum_{T \subseteq A-a_{i}, T \mid=k}\left(\mu\left(T \cup a_{i}\right)-\mu(T)\right) \\
& \mu(A)=1
\end{array}
$$




$$
\begin{aligned}
& \mu(M \cup N)=\mu(M)+\mu(N)+\lambda \mu(M) \mu(N) \\
& \forall M, N \in P(A), M \cap N=\phi \\
& \mu(M) \in[0,1] \quad \forall M \in P(A) \\
& \lambda \in(-1, \infty) \\
& i=1, \ldots, n \text {. }
\end{aligned}
$$

Zhang et al. [39] explained all the parameters in (13) and introduced the calculation methods in detail.

Some simple examples are given to explain how ICMs perform.

3.2.1. Improved Degree Centrality (IDC). In a binary network, $\mathrm{DC}_{i}$ of node $v_{i}$ represents the total number of the connection with node $v_{i}$. However, functional properties of edges, which connect with node $v_{i}$ and node $v_{i}$ cannot be ignored. The improved degree centrality, denoted as $\mathrm{IDC}_{i}$, is given by

$$
\begin{aligned}
T_{t=1, \ldots, N_{i}}\left(e_{i j}\right)= & (c) \int B_{f}\left(e_{i j}\right) d \mu\left(H_{e_{i j}}\right), \\
& \text { if } a_{i j}=1 \text {, exist } e_{i j} \text {; else no exist } e_{i j} \\
H_{1}= & (c) \int\left(T_{1}, \ldots, T_{N_{i}}\right) d \mu\left(H_{T}\right) \\
H_{2}= & (c) \int A_{f}\left(v_{i}\right) d \mu\left(H_{v}\right) \\
\mathrm{IDC}_{i}= & (c) \int\left(\mathrm{DC}_{i}, H_{1}, H_{2}\right) d \mu(H),
\end{aligned}
$$

where $T_{t}\left(e_{i j}\right)$ is the aggregate value of functional properties of edge $e_{i j}$, which is connected to $v_{i}$. The value of $a_{i j}$ is defined as 1 if node $v_{i}$ is connected to $v_{j}$, and 0 otherwise. $H_{1}$ is the aggregate value of functional properties of all edges which are connected to $v_{i} . H_{2}$ is the aggregate value of functional properties of node $v_{i} . \mu$ is the weight of influencing factors.

3.2.2. Improved Closeness Centrality (ICC). The improved closeness centrality of node $v_{i}$, denoted as $\mathrm{ICC}_{i}$, is defined as

$$
\mathrm{ICC}_{i}=\left[\sum_{j}^{N} \mathrm{ISP}_{i j}\right]^{-1},
$$

where $\mathrm{ISP}_{i j}$ is the improved shortest path between nodes $v_{i}$ and $v_{j}$. Its definition and calculation are explained as follows.

Given a directed graph, the length of a path is the number of edges forming it. We define the shortest path as the smallest length among all the paths connecting the source vertex to the target vertex. However, for electromechanical systems, functional properties of nodes and edges also can influence the length of the shortest path. Given that $V_{l_{i}}^{e_{s t}}$ represents a set of nodes, which are in $l_{i}$ th path from node $v_{s}$ to node $v_{t}$, and $E_{l_{i}}^{e_{s t}}$ represents a set of edges, which are in $l_{i}$ th path from node $v_{s}$ to node $v_{t}$, improved shortest path can be expressed as follows:

$$
\begin{aligned}
\operatorname{ISP}_{i j}=\min _{l_{i}} & (c) \int\left\{d_{i j}, Y_{l_{i}}(v), Y_{l_{i}}(e)\right\} d \mu\left(H_{l_{i}}\right) \\
\text { s.t. } \quad & W_{l_{i}}^{e_{s t}}\left(v_{j}\right)=(c) \int A_{f}\left(v_{j}\right) d \mu\left(H_{v_{j}}\right), \\
v_{j} \in V_{l_{i}}^{e_{s t}} & \\
& W_{l_{i}}^{e_{s t}}\left(e_{p q}\right)=(c) \int A_{f}\left(e_{p q}\right) d \mu\left(H_{e_{p q}}\right), \\
& e_{p q} \in E_{l_{i}}^{e_{s t}} \\
& \\
& Y_{l_{i}}(v)=(c) \int\left\{W_{l_{i}}^{e_{s t}}\left(v_{j}\right)\right\} d \mu\left(H_{W_{l_{i}}(v)}\right), \\
& (c) \int\left\{W_{l_{i}}^{e_{s t}}\left(e_{p q}\right)\right\} d \mu\left(V_{l_{i}}^{e_{s t}}\right. \\
&
\end{aligned}
$$

where $W_{l_{i}}^{e_{s t}}\left(v_{j}\right)$ is the aggregate value of functional properties of node $v_{j}$ which is in path $l_{i}$; $W_{l_{i}}^{e_{s t}}\left(e_{p q}\right)$ is the aggregate value of functional properties of edge $e_{p q}$ which is in path $l_{i} ; Y_{l_{i}}(v)$ is the aggregate value of $W_{l_{i}}^{e_{s t}}\left(v_{j}\right)$ which is in path $l_{i} ; Y_{l_{i}}(e)$ is the aggregate value of $W_{l_{i}}^{e_{s t}}\left(e_{p q}\right)$ which is in path $l_{i}$.

3.2.3. Improved Betweenness Centrality (IBC). The improved betweenness centrality of node $v_{i}$, denoted as $\mathrm{IBC}_{i}$, can be rewritten as

$$
\mathrm{IBC}_{i}=\sum_{j, k \neq i} \frac{\operatorname{ISP}_{j k}\left(v_{i}\right)}{\operatorname{ISP}_{j k}}
$$

where ISP ${ }_{j k}$ is the improved shortest path between nodes $v_{j}$ and $v_{k} ; \operatorname{ISP}_{j k}\left(v_{i}\right)$ is the number of those paths that go through node $v_{i}$.

\section{Proposed Method}

The ICMs, such as IDC, ICC, and IBC, reflect the function and structure of system from one aspect and cannot comprehensively reflect the functional and topological characteristics. In some cases, the results of IDC and ICC may be different, even conflicting results. To address this issue, in this paper, comprehensive measure is introduced firstly to explore how to fuse multi-ICMs based on fuzzy integral and then identify influential components. As a well-known fuzzy integral theory, Sugeno integral and Choquet integral have received much interest from researchers and practitioners.

Let us consider a decision matrix $D_{m}=\left(\mathrm{ICM}_{m n}\right)$, where $\mathrm{ICM}_{m n}$ is the $n$th improved centrality measure of node $v_{m}$. 
If we choose Sugeno integral, the comprehensive measure of node $v_{i}$, denoted as IS $i$, is defined as follows:

$$
\begin{aligned}
& \mathrm{IS}_{i}=(s) \int D_{i} d \mu=\bigvee_{j=1}^{m}\left(\mathrm{ICM}_{i(j)} \wedge \mu\left(A_{i(j)}\right)\right), \\
& i=1, \ldots, n .
\end{aligned}
$$

If we choose Choquet integral, the comprehensive measure of node $v_{i}$, denoted as $\mathrm{IC}_{i}$, is defined as follows:

$$
\begin{aligned}
\mathrm{IC}_{i} & =(c) \int D_{i} d \mu \\
& =\sum_{j=1}^{m}\left(\mathrm{ICM}_{i(j)}-\operatorname{ICM}_{i(j-1)}\right) \mu\left(A_{i(j)}\right),
\end{aligned}
$$

$$
i=1, \ldots, n,
$$

where $A_{i(j)}=\left(\mathrm{ICM}_{i(1)}, \ldots, \mathrm{ICM}_{i(N)}\right)$ and $\mu\left(A_{i(j)}\right)$ is the weight for improved centrality measure.

The specific steps of the method are illustrated as follows.

Step 1 (construct the holistic topological network of complex electromechanical system). We can construct a network based on Section 2.1. The components are abstracted as nodes, and the connections between components are represented as edges. And then the system can be described as a network.

Step 2 (calculate properties value of nodes and edges). According to Figure 2, we compute the properties of nodes and edges in combination failure data. The attributes of nodes are obtained by (2) (7), and properties of nodes are computed by (8).

Step 3 (construct and calculate the improved centrality measures by (12)). In this step, we apply (12) to construct and calculate the different improved centrality measures, such as improved degree centrality, improved closeness centrality, and improved betweenness centrality.

Step 4 (select fuzzy integral and fuse all improved centrality measures by (18) or (19)). The alternatives with higher $\mathrm{IC}_{i}$ or $\mathrm{IS}_{i}$ are assumed to be more important and should be given higher priority. Finally, the influence of the node is identified by the value $\mathrm{IC}_{i}$ or IS .

The flow chart of the proposed methods is shown in Figure 3.

\section{A Case Study and Discussion}

China Railway CRHX Size (CRHX) is designed for a speed of $350 \mathrm{~km} / \mathrm{h}$ and each car is suspended by two bogies. The bogie system of the $350 \mathrm{~km} / \mathrm{h}$ EMU train is one of the key parts of CRHX which plays an important role in sustaining the static

\begin{tabular}{|c|c|}
\hline Number & Name \\
\hline$v_{1}$ & Bogie frame \\
\hline$v_{2}$ & Brake Caliper \\
\hline$v_{3}$ & Brake lining \\
\hline$v_{4}$ & Brake discs \\
\hline$v_{5}$ & Booster cylinder \\
\hline$v_{6}$ & Spring \\
\hline$v_{7}$ & Axle box body \\
\hline$v_{8}$ & Vertical shock absorber \\
\hline$v_{9}$ & Bearing \\
\hline$v_{10}$ & Wheel \\
\hline$v_{11}$ & Axle \\
\hline$v_{12}$ & Secondary vertical shock absorber \\
\hline$v_{13}$ & Railway coupling \\
\hline$v_{14}$ & Gearbox \\
\hline$v_{15}$ & Grounding device \\
\hline$v_{16}$ & Traction motor \\
\hline$v_{17}$ & Height adjusting device \\
\hline$v_{18}$ & Antihunting damper \\
\hline$v_{19}$ & Air spring \\
\hline$v_{20}$ & Center pin bush \\
\hline$v_{21}$ & Traction rod \\
\hline$v_{22}$ & Transverse shock absorber \\
\hline$v_{23}$ & Transverse backstop \\
\hline$v_{24}$ & Anti-side-rolling torsion bar \\
\hline$v_{25}$ & Control valve \\
\hline$v_{26}$ & Speed Sensor 1 \\
\hline$v_{27}$ & Speed Sensor 2 \\
\hline$v_{28}$ & LKJ2000 \\
\hline$v_{29}$ & Device for cleaning the tread band of vehicle wheels \\
\hline$v_{30}$ & Acceleration sensor \\
\hline$v_{31}$ & Junction box \\
\hline$v_{32}$ & Temperature sensor bearing \\
\hline$v_{33}$ & Axle temperature sensor \\
\hline$v_{34}$ & AG37 \\
\hline$v_{35}$ & AG43 \\
\hline
\end{tabular}
load from the body weight of a car, carrying the suspensions, brakes, wheels, and axles and controlling wheel sets on curved and straight tracks, in accordance with Figure 4. The bogie
TABLE 1: Components in bogie system.

system can be highly complex due to the systematic use of new technologies and be functional relationship due to the interactions among components. With rapid increase of EMU train speed, the behavior of bogie system becomes more dynamic and uncertain, which not only affects the ride comfort of passengers but also directly relates to the reliability and safety of the train. Generally, the operation of the bogie system depends on 35 components, in accordance with Table 1.

5.1. The Network Model and Related Data. In this section, a case concerning the holistic topological network model of bogie system (as shown in Figure 5) is established as a decision support tool for importance measures and safety assurance, to provide effective support for decision-makers to 


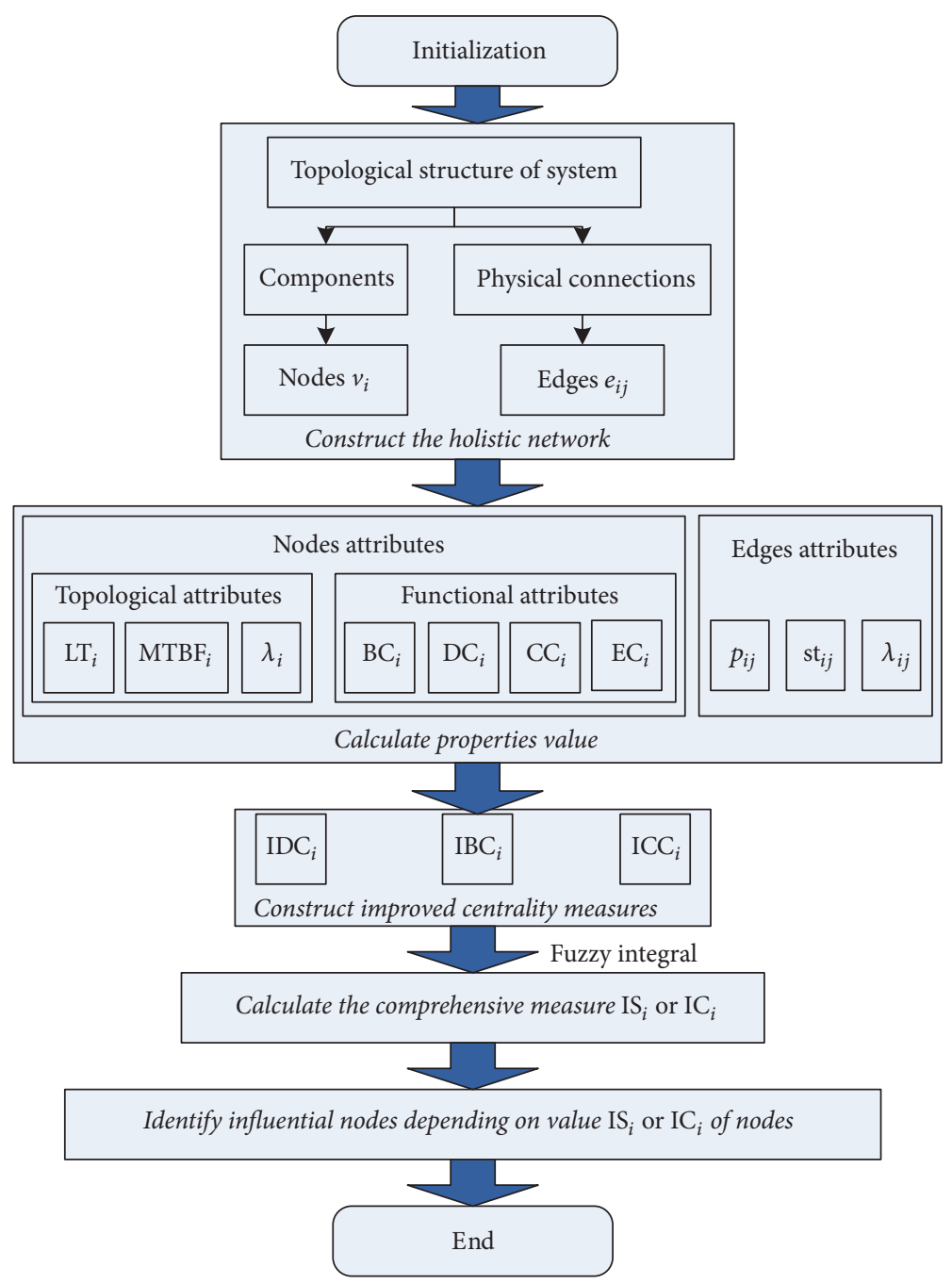

FIgURE 3: The flow chart of the proposed method.
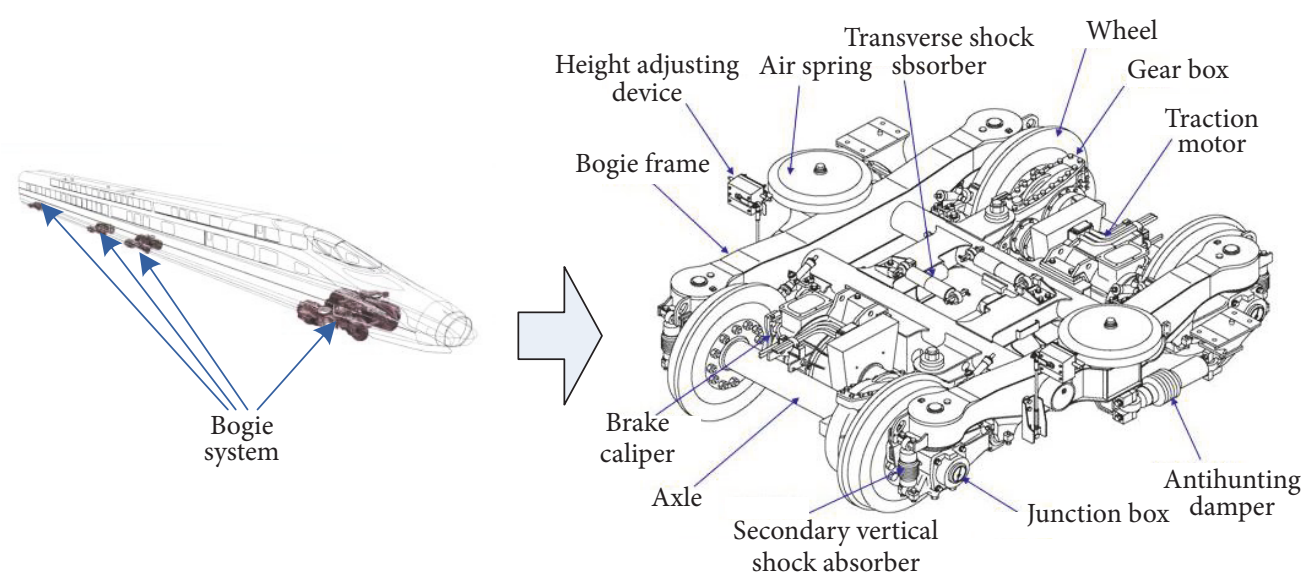

FIGURE 4: Structural features of bogie system. 


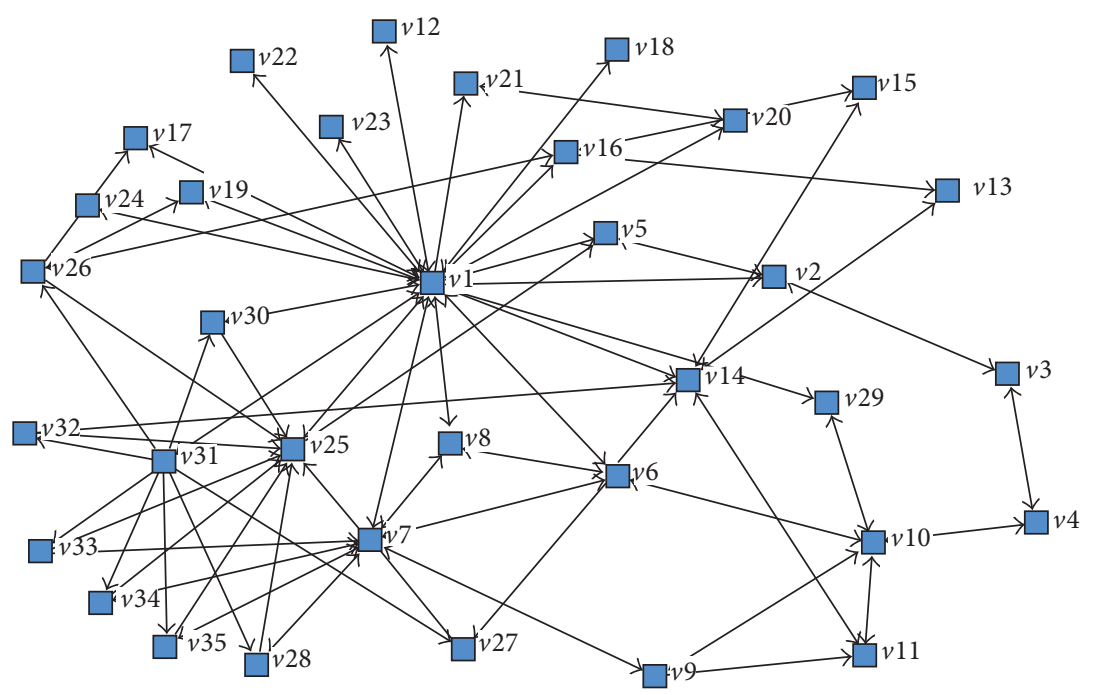

FIGURE 5: The holistic topological network of bogie system: every node $v_{i}$ has property set $\left(A_{f}\left(v_{i}\right), A_{t}\left(v_{i}\right)\right)$, and every edge $e_{i j}$ has property $\operatorname{set}\left(B_{f}\left(e_{i j}\right)\right)$.

proactively understand components interactions and assess their impact to the overall system. According to the construction method of network in Section 2.1, influential factors of bogie system in Figure 2, components of bogie system in Table 1, and the holistic topological network model of bogie system are described as follows:

$$
\begin{aligned}
& \mathrm{HTM}=\langle V, E, f(V), f(E)\rangle \\
& v_{i} \in V=\left\{v_{1}, v_{2}, \ldots, v_{35}\right\}, e_{i j} \in E=\left\{e_{i j} \mid i, j \in 35\right\}, A_{f}\left(v_{i}\right)=\left\{\mathrm{MTBF}_{i}, \lambda_{i}, L_{i}\right\} \subset f(V), A_{t}\left(v_{i}\right)=\left\{\mathrm{DC}_{i}, \mathrm{BC}_{i}, \mathrm{CC}_{i}, \mathrm{EC}_{i}\right\} \subset f(V), B_{f}\left(e_{i j}\right)=\left\{\mathrm{st}_{i j}, \lambda_{i j}, p_{i j}\right\} \subset f(E) .
\end{aligned}
$$

The attributes of edges and nodes in (20) are calculated according to the equation in Section 2.1. Related data, which is necessary for calculation, can be collected from historical failure databases and expert elicitation. For highspeed train system, through a project of cooperation with CRRC Corporation Limited, we have obtained the historical failure databases of CRHX during 2009 to 2014 (863 Program number 2012AA112001). In these databases, each failure data record contains the failure ID numbers, the vehicle ID number, the section of failure, the failure mode, the date of failure, the environment of failure, and so forth. We deal with the data by removing some irrelevant items. However, these data cannot be directly used to compute properties and need to be preprocessed. And, a preprocessed failure data of gear box in bogie system is presented in Table 2 .

Using the preprocessed failure data of CRHX and (2) (3), this paper estimates the parameters for possible faults distribution function. Based on the faults distributing functions and the necessary correction, we can obtain components' failure rate and MTBF. The nodes failure rate and MTBF within 120 million kilometers and service life time are shown in Table 3.

Edges have a striking effect on critical nodes in the network. In essence, edges in holistic topological network also describe components, but these components have different properties from nodes. The fault propagation probability, connection strength, and failure rate of edges are computed according to (2), (8), and (9) based on failure date and shown as in Table 4.

5.2. ICMs for Bogie System. CMs of nodes in the holistic topological network are necessary to construct improved centrality measures to assess influential components. According to (20) and complex network theory, we can obtain centrality measures of all nodes, such as BC, CC, DC, and EC in (4) (7). Figure 6 shows the results of $\mathrm{CMs}$ in the network. We can see that node $v_{1}$ has the highest BC, CC, $\mathrm{DC}$, and EC, and it means that the most important node in the holistic topological network of a bogie system is bogie frame (the corresponding node is $v_{1}$ ). This is consistent with the structural status of the bogie frame in the bogie system.

In the method proposed in this paper, the assessment of influential components requires, firstly, exhaustive and systematic definition and calculation improved centrality measures for any given number of components according to Section 3.2. Figure 7 is presented to demonstrate the calculation results of ICMs by combination with CMs in Figure 5 and functional properties of nodes and edges in Tables 3 and 4. From Figure 7, we can also obtain that bogie frame (the corresponding node is $v_{1}$ ) is identified as the most important components, which is in line with practical experience.

In order to explain the advantages of ICMs, we compare with the identification results of ICMs and CMs. The most 
TABLE 2: The preprocessed failure data of gear box.

\begin{tabular}{|c|c|c|}
\hline Number & Mileage $/ 10^{5} \mathrm{~km}$ & Failure mode \\
\hline (1) & 6.39853 & Oil leakage \\
\hline (2) & 7.87662 & Oil leakage \\
\hline (3) & 7.90238 & Gear shift \\
\hline (4) & 10.02856 & Oil leakage \\
\hline (5) & 11.26585 & Crackle \\
\hline (6) & 11.29788 & Oil leakage \\
\hline (7) & 12.39568 & Oil leakage \\
\hline (8) & 14.02572 & Crackle \\
\hline (9) & 15.64292 & Crackle \\
\hline (10) & 16.39853 & Oil leakage \\
\hline (11) & 16.64292 & Crackle \\
\hline (12) & 16. 64292 & Oil leakage \\
\hline (13) & 17.66824 & Oil leakage \\
\hline (14) & 18.16762 & Gear shift \\
\hline (15) & 18.2579 & Oil leakage \\
\hline (16) & 19.32587 & Gear shift \\
\hline (17) & 19.90225 & Oil leakage \\
\hline (18) & 21.0191 & Crackle \\
\hline (19) & 22.17948 & Crackle \\
\hline (20) & 22.38788 & Crackle \\
\hline$(21)$ & 23.0191 & Oil leakage \\
\hline (22) & 27.73312 & Oil leakage \\
\hline (23) & 28.62366 & Crackle \\
\hline$(24)$ & 28.87598 & Oil leakage \\
\hline (25) & 29.62366 & Oil leakage \\
\hline (26) & 31.79775 & Crackle \\
\hline (27) & 32.4842 & Crackle \\
\hline (28) & 33.43831 & Crackle \\
\hline (29) & 34.43831 & Gear shift \\
\hline$(30)$ & 34.50838 & Crackle \\
\hline (31) & 37.66297 & Oil leakage \\
\hline (32) & 38.66297 & Gear shift \\
\hline (33) & 40.0016 & Crackle \\
\hline
\end{tabular}

critical node in bogie system is all $v_{1}$. However, the importance ranking of other components is also the key to ensure system safety and reliability. In fact, maintenance personnel give more attention to a series of critical components, but not only the most important component. Figure 8 indicates the ranking of all nodes. We can clearly find that $v_{2}$, which is the identification result by using DC, is more important in network from a topological point of view. However, if we use improved DC to analyze the Brake Caliper, the result shows that Brake Caliper is not much more important than other nodes. This effect can be explained by the fact that ICMs of components are influenced by functional properties of nodes and edges that are linked to closer neighbors at the same time. Hence, with the functional influence of nodes and edges, the importance of nodes may change. Just as we have mentioned, ICMs is better than CMs.

Another interesting fact observed is that, as presented in Figure 8, the ranking of all nodes is different by using IBC,
TABLE 3: The nodes functional properties.

\begin{tabular}{|c|c|c|c|}
\hline Node & Life time/year & Failure rate & MTBF \\
\hline$v_{1}$ & 20 & 0.0134 & 2.34 \\
\hline$v_{2}$ & 20 & 0.00798 & 1.25 \\
\hline$v_{3}$ & 15 & 0.0089 & 1.54 \\
\hline$v_{4}$ & 20 & 0.0045 & 2.21 \\
\hline$v_{5}$ & 20 & 0.0079 & 1.72 \\
\hline$v_{6}$ & 20 & 0.0059 & 1.92 \\
\hline$v_{7}$ & 20 & 0.0086 & 1.41 \\
\hline$v_{8}$ & 20 & 0.0081 & 1.69 \\
\hline$v_{9}$ & 20 & 0.0144 & 1.21 \\
\hline$v_{10}$ & 20 & 0.0126 & 1.34 \\
\hline$v_{11}$ & 20 & 0.0176 & 1.47 \\
\hline$v_{12}$ & 20 & 0.0079 & 1.65 \\
\hline$v_{13}$ & 20 & 0.0082 & 1.41 \\
\hline$v_{14}$ & 20 & 0.0103 & 1.52 \\
\hline$v_{15}$ & 20 & 0.0103 & 1.81 \\
\hline$v_{16}$ & 20 & 0.0078 & 1.45 \\
\hline$v_{17}$ & 20 & 0.0116 & 1.44 \\
\hline$v_{18}$ & 20 & 0.0082 & 1.66 \\
\hline$v_{19}$ & 15 & 0.0061 & 2.03 \\
\hline$v_{20}$ & 20 & 0.0052 & 1.55 \\
\hline$v_{21}$ & 20 & 0.0051 & 1.97 \\
\hline$v_{22}$ & 20 & 0.0062 & 1.86 \\
\hline$v_{23}$ & 20 & 0.0049 & 1.93 \\
\hline$v_{24}$ & 20 & 0.0051 & 1.77 \\
\hline$v_{25}$ & 20 & 0.0072 & 1.78 \\
\hline$v_{26}$ & 20 & 0.0077 & 1.68 \\
\hline$v_{27}$ & 20 & 0.0177 & 1.32 \\
\hline$v_{28}$ & 20 & 0.0187 & 1.43 \\
\hline$v_{29}$ & 20 & 0.0107 & 1.50 \\
\hline$v_{30}$ & 20 & 0.0152 & 1.41 \\
\hline$v_{31}$ & 20 & 0.0049 & 1.53 \\
\hline$v_{32}$ & 20 & 0.0165 & 1.32 \\
\hline$v_{33}$ & 15 & 0.0189 & 1.38 \\
\hline$v_{34}$ & 20 & 0.0191 & 1.49 \\
\hline$v_{35}$ & 20 & 0.0169 & 1.53 \\
\hline
\end{tabular}

ICC, IDC, and IEC. Note that the result of IDC shows that $v_{1}$, traction motor $v_{16}$, and $v_{7}$ are the critical components. If we use ICC to identify influential components, the results reveal that $v_{1}, v_{7}$, and $v_{6}$ are the most important for bogie system. However, $v_{1}, v_{7}$, and $v_{14}$ are the critical components by using IBC identification, and the identification results of IEC show that $v_{7}, v_{25}$, and $v_{31}$ are essential for bogie system. This research presented two aspects of reasons for this: one is different influences which are taken into consideration and another is the uncertainty and randomness when single measure is used to identify critical component. For example, $\mathrm{DC}_{2}<\mathrm{IDC}_{2}$; however, $\mathrm{CC}_{2}>\mathrm{ICC}_{2} ; \mathrm{DC}_{15}<\mathrm{IDC}_{15}$, but $\mathrm{BC}_{15}>\mathrm{IBC}_{15}$. CMs are constructed in which topological properties are taken into consideration, and ICMs are defined in combination with functional and topological features. 
TABLE 4: Functional properties of several edges.

\begin{tabular}{|c|c|c|c|c|c|c|c|}
\hline Edge & $\begin{array}{l}\text { Connection } \\
\text { strength }\end{array}$ & Failure rate & $\begin{array}{c}\text { Fault } \\
\text { propagation } \\
\text { probability }\end{array}$ & Edge & $\begin{array}{l}\text { Connection } \\
\text { strength }\end{array}$ & Failure rate & $\begin{array}{c}\text { Fault } \\
\text { propagation } \\
\text { probability }\end{array}$ \\
\hline$e_{12}$ & 0.4 & 0.0051 & 0.0001 & $e_{1,14}$ & 0.6 & 0.0068 & 0.0003 \\
\hline$e_{13}$ & 0.5 & 0.0087 & 0.0005 & $e_{51}$ & 0.4 & 0.0053 & 0.0004 \\
\hline$e_{15}$ & 0.5 & 0.0095 & 0.0006 & $e_{61}$ & 0.7 & 0.0071 & 0.0001 \\
\hline$e_{16}$ & 0.8 & 0.0088 & 0.0003 & $e_{52}$ & 0.5 & 0.0067 & 0.0015 \\
\hline$e_{17}$ & 0.6 & 0.0082 & 0.0003 & $e_{67}$ & 0.3 & 0.0104 & 0.0010 \\
\hline$e_{18}$ & 0.5 & 0.0062 & 0.0007 & $e_{10,4}$ & 0.7 & 0.0045 & 0.0013 \\
\hline$e_{1,12}$ & 0.7 & 0.0079 & 0.0004 & $e_{11,9}$ & 0.4 & 0.0042 & 0.0011 \\
\hline$\cdots$ & $\cdots$ & $\cdots$ & $\ldots$ & $\cdots$ & $\cdots$ & $\cdots$ & $\ldots$ \\
\hline
\end{tabular}
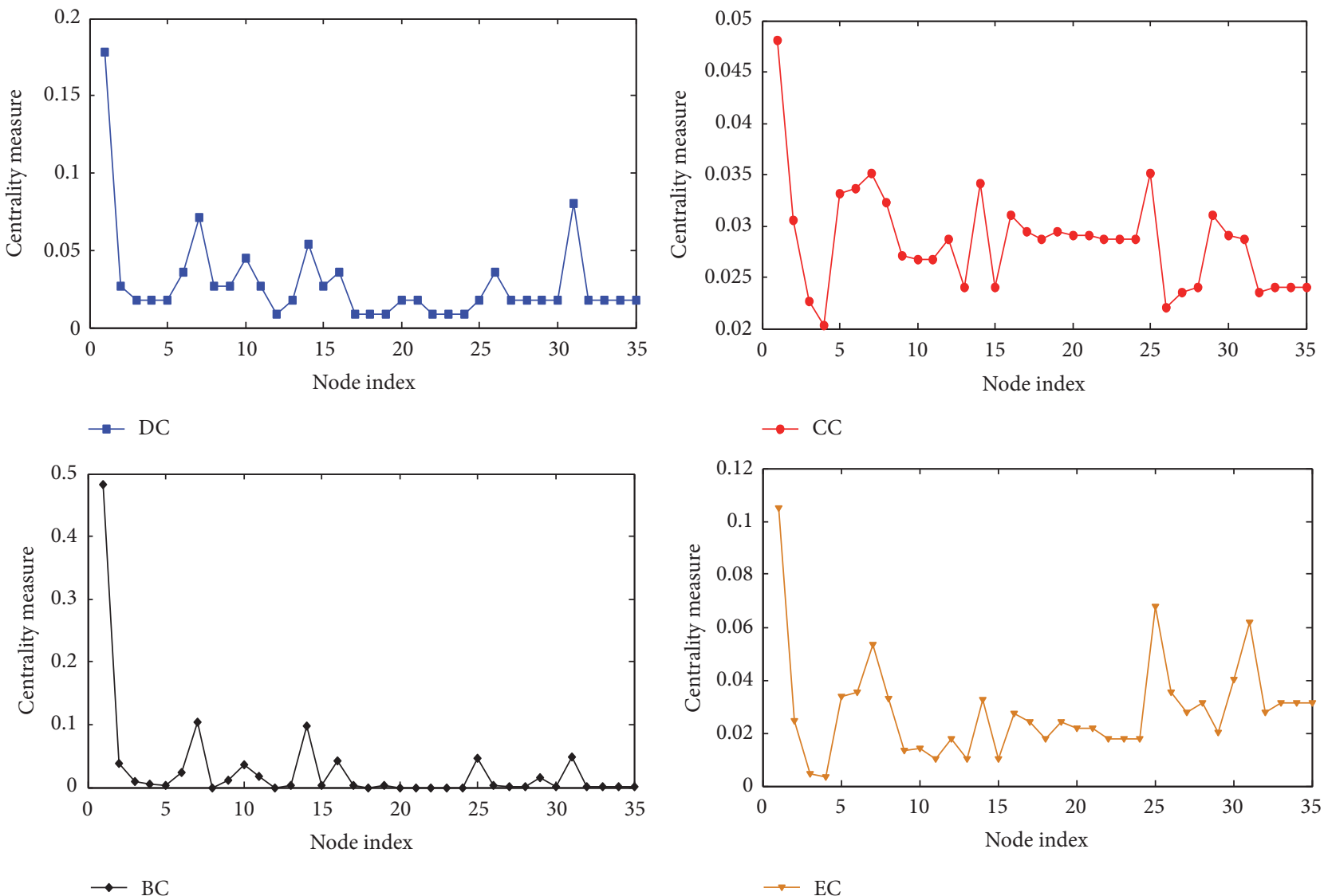

Figure 6: CMs of nodes in the holistic topological network.

5.3. Influential Components Based on Fuzzy Integral. In order to overcome the uncertainty and randomness which applies for single measure identifying critical component, we fuse ICMs to explore how to identify influential components based on fuzzy integral. According to Section 4, Figure 9 is described to demonstrate the result of comprehensive importance calculations. The result shows that the bogie frame and axle box body (the corresponding nodes are $v_{1}, v_{7}$ ) are identified as the most important components, which is in line with practical experience. The more critical nodes which are identified by Sugeno integral are the same as the Choquet integral. However, the calculation of Choquet integral is smaller than Sugeno integral and there is a problem that Sugeno integral will miss the information in calculation process. Therefore, the different integrals are selected based on different operation conditions when only more critical components need to be evaluated.

Meanwhile, how to calculate weight $\mu$ in fuzzy integral also is important for the accuracy of assessment results. The two methods are provided to compute weight $\mu$, and 

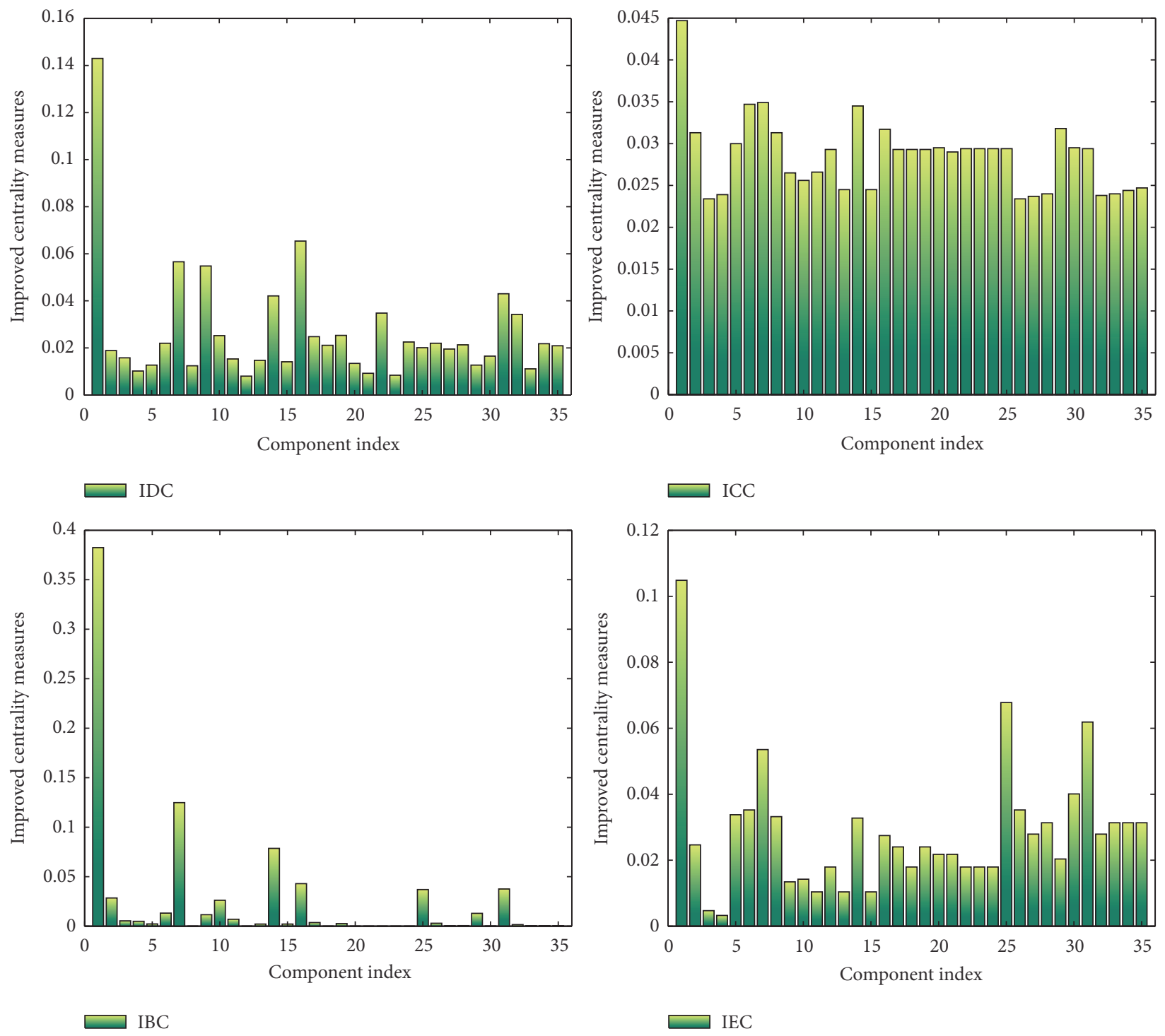

FIGURE 7: Improved centrality measures.

they are $g$ fuzzy measure and $k$ additive fuzzy measure. Theoretically, the accuracy of computation results based on $k$ additive fuzzy measure is higher than $g$ fuzzy measure. $g$ fuzzy measure analyzes weights of all influences and their influencing relationship; however, $k$ additive fuzzy measure provides weights of all influences and their influencing relationship for each nodes. Moreover, the application of $k$ additive fuzzy measure is limited to the problem of large quantities of computation. Fortunately, we can find that most identification results with $g$ fuzzy measure are the same as $k$ additive fuzzy measure in Figure 9. Therefore, the $g$ fuzzy measure can be chosen in traditional study.

5.4. Discussion. To better assess the importance of a component in the holistic topological network, the method of integration ICMs is proposed in this paper, which takes functional and topological properties into account. It is much different from integration topological CMs indicators such as DC, BC, CC, and EC mentioned in Section 5.2. From an experimental viewpoint, Figure 10 shows the results of integration CMs and ICMs. The ranking of all nodes by comprehensive measure, which is constructed by integration ICMs, is different from integration CMs. The weakness and shortage of CMs cannot disappear if we integrate CMs. Therefore, the accuracy of only integration CMs is still lower.

Table 5 presents the evaluation results of different methods. Based on 863 Program (number 2012AA112001), we investigate the bogie system of CRHX in-depth, communicate with maintenance personnel, and attain the ranking of important components for using the enterprise, as shown in Practical recognition in Table 5. From the expert experience viewpoint, the most important nodes in the holistic topological network of a bogie system are $v_{1}, v_{7}, v_{16}$, and $v_{14}$, respectively. In the view of ICMs, these centrality measures 

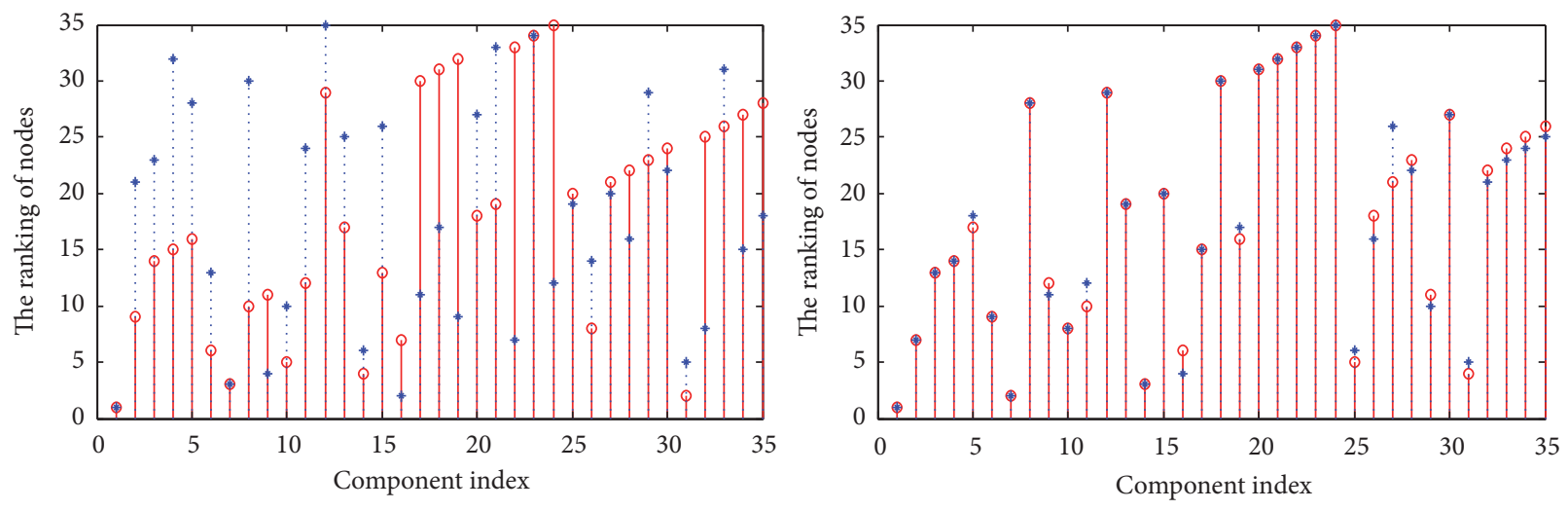

$\begin{array}{ll}\rightarrow & \text { DC } \\ \cdots & *\end{array}$

$\rightarrow$ BC
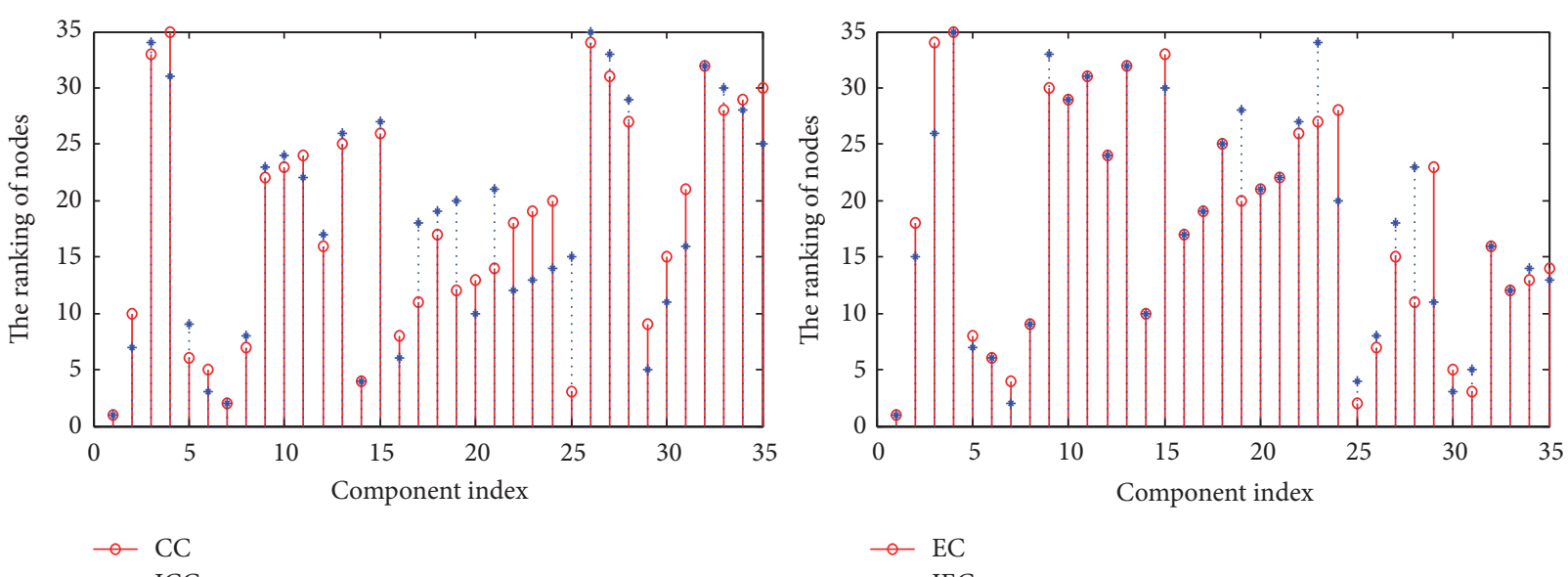

Figure 8: The ranking of nodes.

TABLE 5: The evaluation results of different methods.

\begin{tabular}{lcccccccccccc}
\hline Number & AHP & TOPSIS & DC & CC & BC & EC & Practical recognition & \multicolumn{2}{c}{ Sugeno integral } & \multicolumn{2}{c}{ Choquet integral } \\
& & & & & & & $g_{\lambda}$ & $k$-additive & $g_{\lambda}$ & $k$-additive \\
\hline$(1)$ & $v_{1}$ & $v_{1}$ & $v_{1}$ & $v_{1}$ & $v_{1}$ & $v_{1}$ & $v_{1}$ & $v_{1}$ & $v_{1}$ & $v_{1}$ & $v_{1}$ \\
$(2)$ & $v_{16}$ & $v_{7}$ & $v_{31}$ & $v_{7}$ & $v_{7}$ & $v_{25}$ & $v_{7}$ & $v_{7}$ & $v_{7}$ & $v_{7}$ & $v_{7}$ \\
$(3)$ & $v_{14}$ & $v_{14}$ & $v_{7}$ & $v_{25}$ & $v_{14}$ & $v_{31}$ & $v_{14}$ & $v_{6}$ & $v_{14}$ & $v_{14}$ & $v_{14}$ \\
$(4)$ & $v_{31}$ & $v_{9}$ & $v_{14}$ & $v_{14}$ & $v_{31}$ & $v_{7}$ & $v_{16}$ & $v_{14}$ & $v_{16}$ & $v_{16}$ & $v_{16}$ \\
$(5)$ & $v_{7}$ & $v_{16}$ & $v_{10}$ & $v_{6}$ & $v_{25}$ & $v_{30}$ & $v_{31}$ & $v_{29}$ & $v_{31}$ & $v_{9}$ & $v_{9}$ \\
$(6)$ & $v_{9}$ & $v_{8}$ & $v_{6}$ & $v_{5}$ & $v_{16}$ & $v_{6}$ & $v_{9}$ & $v_{16}$ & $v_{25}$ & $v_{31}$ & $v_{31}$ \\
$(7)$ & $v_{19}$ & $v_{12}$ & $v_{16}$ & $v_{8}$ & $v_{2}$ & $v_{26}$ & $v_{25}$ & $v_{2}$ & $v_{2}$ & $v_{32}$ & $v_{25}$ \\
$(8)$ & $v_{25}$ & $v_{23}$ & $v_{26}$ & $v_{16}$ & $v_{10}$ & $v_{5}$ & $v_{6}$ & $v_{8}$ & $v_{10}$ & $v_{6}$ & $v_{6}$ \\
$(9)$ & $v_{2}$ & $v_{22}$ & $v_{2}$ & $v_{29}$ & $v_{6}$ & $v_{8}$ & $v_{32}$ & $v_{5}$ & $v_{6}$ & $v_{25}$ & $v_{22}$ \\
$(10)$ & $v_{10}$ & $v_{21}$ & $v_{8}$ & $v_{2}$ & $v_{11}$ & $v_{14}$ & $v_{22}$ & $v_{20}$ & $v_{29}$ & $v_{22}$ & $v_{32}$ \\
\hline
\end{tabular}

can be further extended by considering suitable properties to identify the important components with respect to function and topology of the nodes and edges (in Figure 6), while, using fuzzy integral fusion ICMs, some nodes (such as $v_{6}$ ) with low values can be also identified as the most important nodes. In addition, the evaluation result of these methods is a larger gap, especially noncritical components. These methods, such as AHP and TOPSIS, are greatly affected by human factors. The accuracy of the most critical components identification results is higher, but other components have great difference on human factors. CMs, which include DC, $\mathrm{CC}, \mathrm{BC}$, and EC, identify the critical components of structure. 

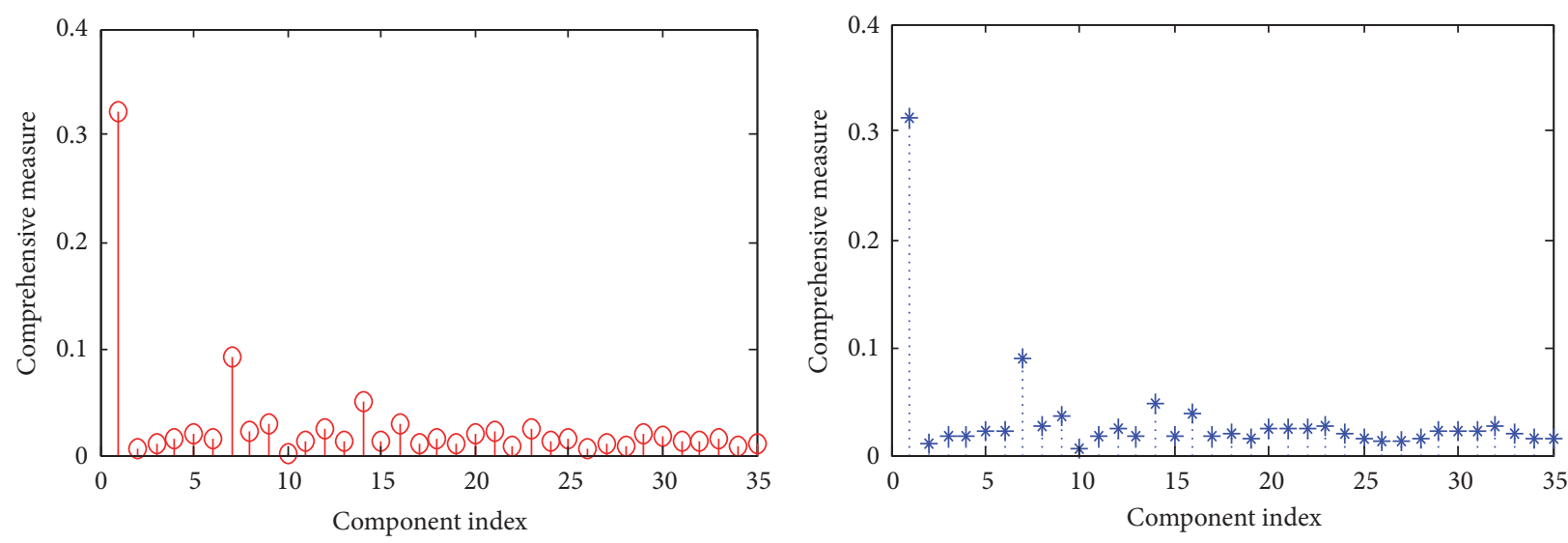

- Choquet integral_g
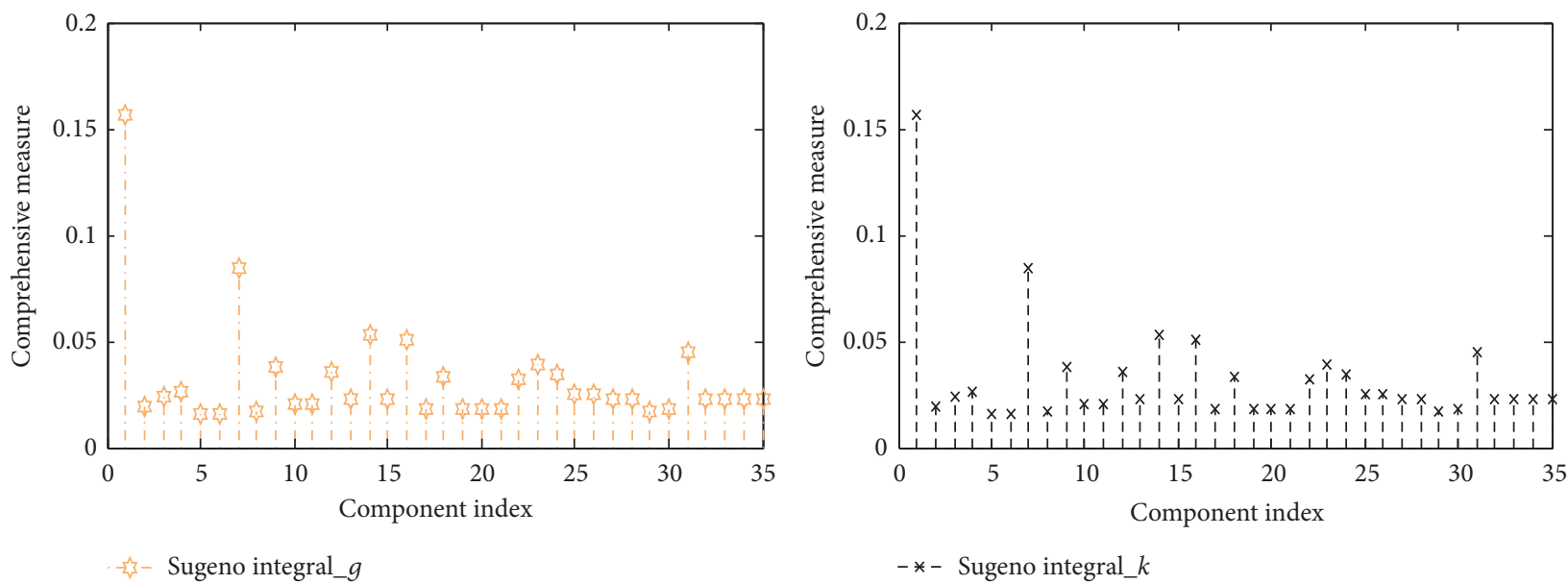

FIGURE 9: Influential components of bogie system.

Therefore, there are some differences in the results of CMs and other measures. In fact, there are still differences of using Sugeno integral and Choquet integral to identify influential components. The most important components are the same, while the ranking of noncritical components is different. This is because the amount of information loss exceeds the threshold value.

With the reduction of the importance of nodes, the difference of all methods in Table 5 for importance of nodes is bigger in Figure 11. For example, the results of the three methods, such as AHP, DC, and EC, are not consistent with practical recognition for node $v_{7}$. And, for nodes $v_{14}, v_{16}$, and $v_{31}$, the number of methods which are not different from practical recognition, is 4,7 , and 9 , respectively. We can see that most methods are effective in identifying the most critical nodes. However, the validity of the identification results of the other nodes is poor.

The methods of integration multimeasures are able to overcome the randomness and uncertainty by using a single measure. Different methods of comprehensive multimeasures are selected, and the accuracy of the identification results is different. Figure 12 presents the accuracy rate of these methods in comparison with practical recognition. The accuracy rate $\mathrm{AR}_{i}=\mathrm{RN}_{i} / N$, where $\mathrm{RN}_{i}$ indicates the number of nodes in the $i$ th method which the ranking is consistent with the expert experience, and $N_{i}$ is the number of nodes in system. We can find that Choquet integral by using $k$ additive has a higher accuracy rate. The method of integration ICMs with Sugeno integral misses much information in computational procedure. If the amount of information loss exceeds the threshold value, the results will be not accurate.

Through the exploratory discussion above, it is shown that the results acquired from comprehensive measure are, to a certain extent, more reasonable and powerful than those traditional importance measures. Just as mentioned, the system function represents the interactions between components; system topology represents the structure relationship. Therefore, the comprehensive gives consideration to both topological features and physical characteristics of a holistic topological network from multiple perspectives.

\section{Conclusions}

This paper integrates the literature of mechatronic architecture and complex networks to define holistic topological 

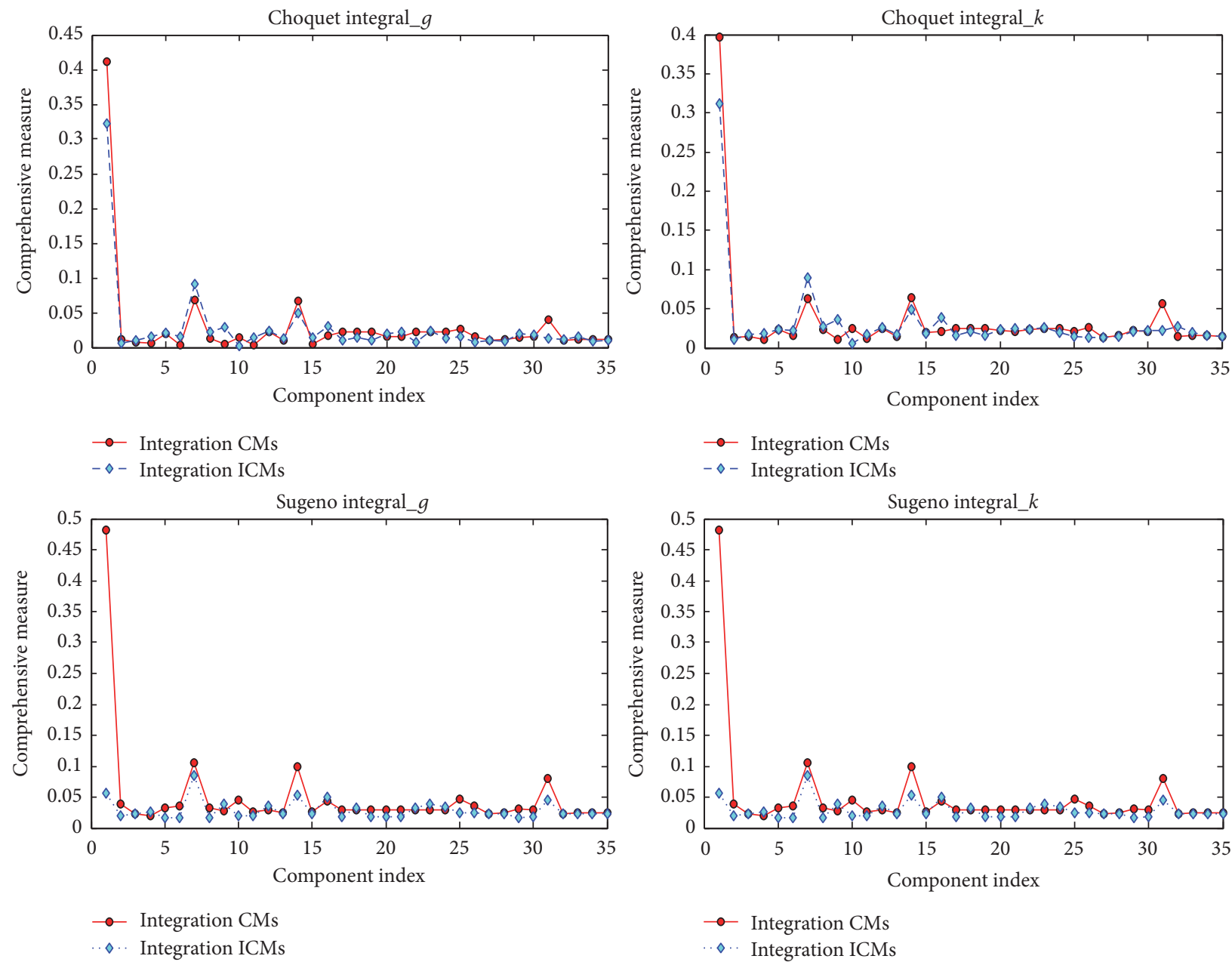

Figure 10: The results of integration CMs and ICMs.

network. And, based on the notion of complex networks, meaningful improved centrality measures (ICMs) are first brought and then comprehensive measure, with first-time, is constructed to identify important components by integrated multi-ICMs. Indeed, construction ICMs with the consideration of functional and topological properties and their relationship is the originality and novelty of proposed measures. Next, integration multi-ICMs based on fuzzy integral, that is, the combination of multiple influencing factors, is also the novelty of comprehensive measure. This paper has also shown the application of the proposed approach in reliability assessment of bogie system of CRHX EMUs. By applying the comprehensive measure, the components importance of bogie system can be evaluated at reasonable human factors. Results indicate that the ranking of critical components can be not the same in selecting different fuzzy integrals. According to the applicable environment, reasonable choices can be determined. In addition, three conclusions are drawn through an exploratory discussion: (i) The function and topology are of the same importance in electromechanical system. If identifying critical components, these two aspects should be taken into account.

(ii) The method of integration ICMs with Choquet integral by using $k$-additive has a higher accuracy rate than other methods.

(iii) The result of comprehensive evaluation is better than that of single measure identification.

Of course, due to the diversification and complexity of the real electromechanical system, the model presented here is just a simplification of what happens in actual systems. Several influential factors of critical components in the model need to be further developed if some additional information can be acquired. As previously mentioned, the robustness of comprehensive measure with respect to the fault propagation damping parameter is still under discussion and valuable for further research. 

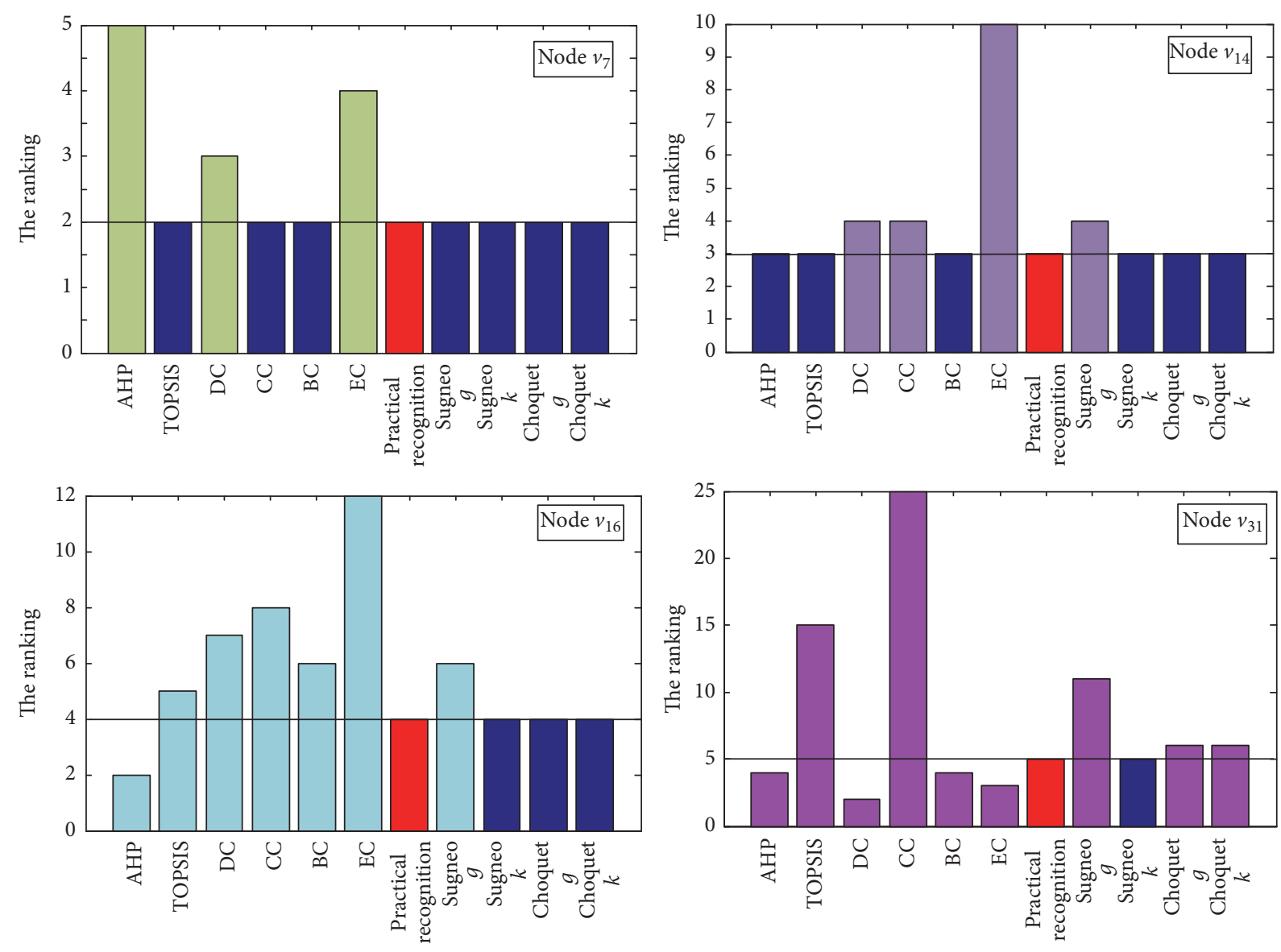

Figure 11: The ranking of partly nodes.

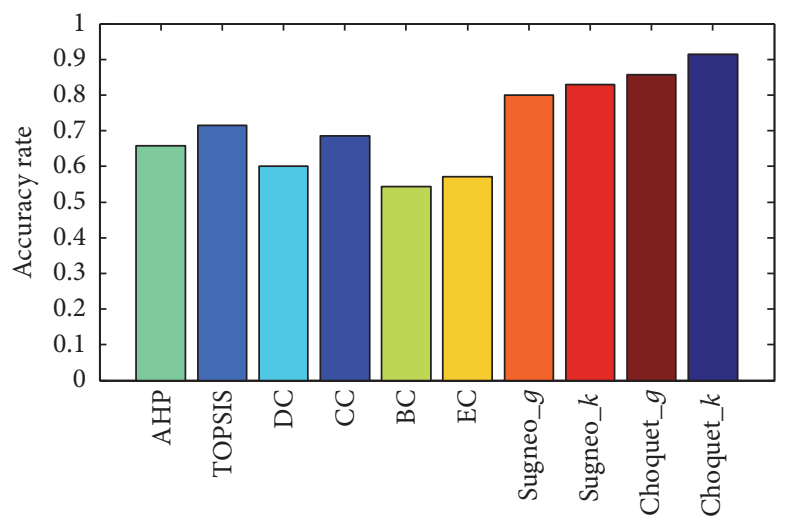

FIgURE 12: The accuracy rate of these methods.

\section{Conflicts of Interest}

The authors declare that there are no conflicts of interest regarding the publication of this paper.

\section{Acknowledgments}

The work is partially supported by the State Key Laboratory of Rail Traffic Control and Safety, Beijing Jiaotong University,
Beijing, China (Grant no. RCS2016ZZ002), and the National Key Research and Development Program of China (Grant no. 2016YFB1200402).

\section{References}

[1] W. E. Vesely, F. F. Goldberg, N. H. Roberts, and D. F. Haasl, Fault tree handbook. No. NUREG-0492, Nuclear Regulatory Commission, Washington, DC, USA, 1981. 
[2] X. Zhu and W. Kuo, "Importance measures in reliability and mathematical programming," Annals of Operations Research, vol. 212, pp. 241-267, 2014.

[3] L. W. Birnbaum, "On the importance of different elements in a multi-element system," Multivariate Analysis, vol. 2, 1969.

[4] J. B. Fussell, "How to hand-calculate system reliability and safety characteristics," IEEE Transactions on Reliability, vol. 3, pp. 169174, 1975.

[5] E. A. Elsayed, Reliability engineering, Addison Wesley Longman, 1996.

[6] J. E. Ramirez-Marquez and W. C. David, "Composite importance measures for multi-state systems with multi-state components," Reliability, IEEE Transactions on, vol. 54, no. 3, pp. 517529, 2005.

[7] A.-L. Barabsi and R. Albert, "Emergence of scaling in random networks," Science, vol. 286, no. 5439, pp. 509-512, 1999.

[8] S. H. Strogatz, "Exploring complex networks," Nature, vol. 410, no. 6825 , pp. 268-276, 2001.

[9] D. Wei, X. Deng, X. Zhang, Y. Deng, and S. Mahadevan, "Identifying influential nodes in weighted networks based on evidence theory," Physica A: Statistical Mechanics and Its Applications, vol. 392, no. 10, pp. 2564-2575, 2013.

[10] D. J. Brass and E. B. Marlene, "Centrality and power in organizations," Networks and Organizations: Structure, Form, and Action, vol. 191, p. 215, 1992.

[11] L. C. Freeman, "A set of measures of centrality based on betweenness," Sociometry, vol. 40, no. 1, pp. 35-41, 1977.

[12] P. Bonacich and L. Paulette, "Eigenvector Centrality and Structural Zeroes and Ones: When Is a Neighbor Not a Neighbor?" Social Networks, vol. 43, pp. 86-90, 2015.

[13] B. Dan, L. Li, X. Zhang, F. Guo, and J. Zhou, "Networkintegrated manufacturing system," International Journal of Production Research, vol. 43, no. 12, pp. 2631-2647, 2005.

[14] Y. Li, X. Chu, D. Chu, and Q. Liu, "An integrated module partition approach for complex products and systems based on weighted complex networks," International Journal of Production Research, vol. 52, no. 15, pp. 4608-4622, 2014.

[15] H.-Q. Jiang, J.-M. Gao, F.-M. Chen, and Z.-Y. Gao, "Vulnerability analysis to distributed and complex electromechanical system based on network property," Computer Integrated Manufacturing Systems, vol. 15, no. 4, pp. 791-796, 2009.

[16] D. G. Xu, Y. Q. Gui, and P. L. Zhao, "Research on reliability of the multi-effect alumina evaporation system based on networks cascading failure model," in Proceedings of the Control Conference (CCC, 2013 32nd Chinese), pp. 8363-8368, IEEE, 2013.

[17] G. Zong, C. Zhang, and W. Liu, "Study on complexities in relational network of component maintenance for high-speed train in network perspective," China Railway Science, vol. 34, no. 3, pp. 105-108, 2013.

[18] T. Gwo-Hshiung, G. H. Tzeng, and J.-J. Huang, Multiple Attribute Decision Making: Methods and Applications, CRC Press, 2011.

[19] G. Büyüközkan and S. Güleryüz, "Multi Criteria Group Decision Making Approach for Smart Phone Selection Using Intuitionistic Fuzzy TOPSIS," International Journal of Computational Intelligence Systems, vol. 9, no. 4, pp. 709-725, 2016.

[20] D. Joshi and S. Kumar, "Interval-valued intuitionistic hesitant fuzzy Choquet integral based TOPSIS method for multi-criteria group decision making," European Journal of Operational Research, vol. 248, no. 1, pp. 183-191, 2016.
[21] M. A. Sharaf and H. A. Helmy, "A classification model for inventory management of spare parts," in Proceedings of the International conference on production, industrial engineering, vol. 7, 2001.

[22] X. Liu and S. An, "Failure propagation analysis of aircraft engine systems based on complex network," Procedia Engineering, vol. 80, pp. 506-521, 2014.

[23] F. Jin, L. Pei, H. Chen, and L. Zhou, "Interval-valued intuitionistic fuzzy continuous weighted entropy and its application to multi-criteria fuzzy group decision making," Knowledge-Based Systems, vol. 59, pp. 132-141, 2014.

[24] K. Govindan, S. Rajendran, J. Sarkis J, and Murugesan., "Multi criteria decision making approaches for green supplier evaluation and selection: a literature review," Journal of Cleaner Production, vol. 98, pp. 66-83, 2015.

[25] E. Zio and L. R. Golea, "Analyzing the topological, electrical and reliability characteristics of a power transmission system for identifying its critical elements," Reliability Engineering and System Safety, vol. 101, pp. 67-74, 2012.

[26] Y. Du, C. Gao, Y. Hu, S. Mahadevan, and Y. Deng, "A new method of identifying influential nodes in complex networks based on TOPSIS," Physica A: Statistical Mechanics and its Applications, vol. 399, pp. 57-69, 2014.

[27] F. Papadopoulos, C. Psomas, and D. Krioukov, "Replaying the geometric growth of complex networks and application to the as internet," ACM SIGMETRICS Performance Evaluation Review, vol. 40, no. 3, pp. 104-106, 2012.

[28] C. Gan, X. Yang, W. Liu, Q. Zhu, J. Jin, and L. He, "Propagation of computer virus both across the Internet and external computers: a complex-network approach," Communications in Nonlinear Science and Numerical Simulation, vol. 19, no. 8, pp. 2785-2792, 2014.

[29] O. Sporns, "Network attributes for segregation and integration in the human brain," Current Opinion in Neurobiology, vol. 23, no. 2, pp. 162-171, 2013.

[30] D. R. Carter, L. A. DeChurch, M. T. Braun, and N. S. Contractor, "Social network approaches to leadership: An integrative conceptual review," Journal of Applied Psychology, vol. 100, no. 3, pp. 597-622, 2015.

[31] U. Brandes, S. P. Borgatti, and L. C. Freeman, "Maintaining the duality of closeness and betweenness centrality," Social Networks, vol. 44, pp. 153-159, 2016.

[32] S. Uddin, H. Liaquat, and W. T. Rolf, "New direction in degree centrality measure: Towards a time-variant approach," International Journal of Information Technology \& Decision Making, vol. 13, no. 4, pp. 865-878, 2014.

[33] J. Gao, G. Li, and Z. Gao, "Fault propagation analysis for complex system based on small-world network model," in Proceedings of the 54th Annual Reliability and Maintainability Symposium, RAMS 2008, IEEE, January 2008.

[34] E. M. Daly and M. Haahr, "Social network analysis for information flow in disconnected delay-tolerant MANETs," IEEE Transactions on Mobile Computing, vol. 8, no. 5, pp. 606-621, 2009.

[35] Y. Wang, L. Bi, S. Lin, M. Li, and H. Shi, "A complex networkbased importance measure for mechatronics systems," Physica A: Statistical Mechanics and its Applications, vol. 466, pp. 180198, 2017.

[36] M. Sugeno, Theory of fuzzy integrals and its applications [Ph.D. thesis], Tokyo Institute of Technology, Tokyo, Japan, 1974. 
[37] M. Sugeno, "Fuzzy measures and fuzzy integrals: a survey," in Fuzzy Automata and Decision Processes, Gupta, Saridis, and Gaines, Eds., vol. 89, p. 102, 1977.

[38] J. J. Liou, Y.-C. Chuang, and G.-H. Tzeng, "A fuzzy integralbased model for supplier evaluation and improvement," Information Sciences, vol. 266, pp. 199-217, 2014.

[39] L. Zhang, D.-Q. Zhou, P. Zhou, and Q.-T. Chen, "Modelling policy decision of sustainable energy strategies for Nanjing city: a fuzzy integral approach," Renewable Energy, vol. 62, pp. 197203, 2014.

[40] J. Zhai, H. Xu, and Y. Li, "Fusion of extreme learning machine with fuzzy integral," International Journal of Uncertainty, Fuzziness and Knowledge-Based Systems, vol. 21, supplement 2, no. December 2013, pp. 23-34, 2013.

[41] M. F. Anderson, D. T. Anderson, and D. J. Wescott, "Estimation of adult skeletal age-at-death using the sugeno fuzzy integral," American Journal of Physical Anthropology, vol. 142, no. 1, pp. 30-41, 2010.

[42] E. E. Karsak and M. Dursun, "An integrated fuzzy MCDM approach for supplier evaluation and selection," Computers and Industrial Engineering, vol. 82, pp. 82-93, 2015.

[43] T. Murofushi and M. Sugeno, "An interpretation of fuzzy measures and the Choquet integral as an integral with respect to a fuzzy measure," Fuzzy Sets and Systems, vol. 29, no. 2, pp. 201-227, 1989.

[44] M. Grabisch, "The application of fuzzy integrals in multicriteria decision making," European Journal of Operational Research, vol. 89, no. 3, pp. 445-456, 1996.

[45] J.-L. Marichal and M. Roubens, "Entropy of discrete fuzzy measures," International Journal of Uncertainty, Fuzziness and Knowledge-Based Systems, vol. 8, no. 6, pp. 625-640, 2000. 


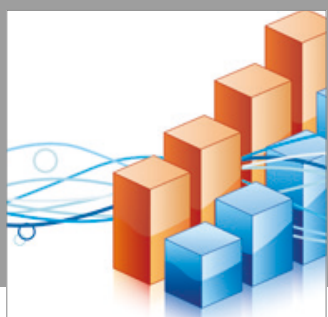

Advances in

Operations Research

vatersals

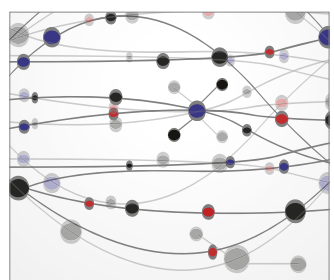

\section{The Scientific} World Journal
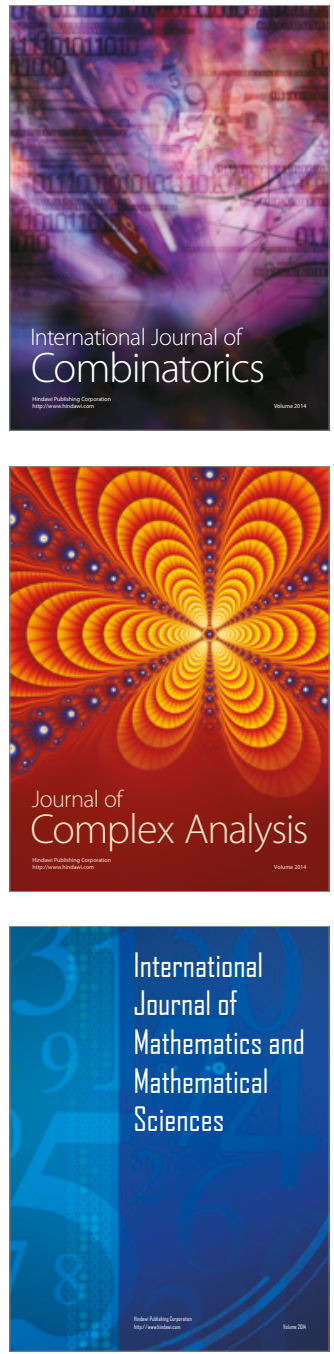
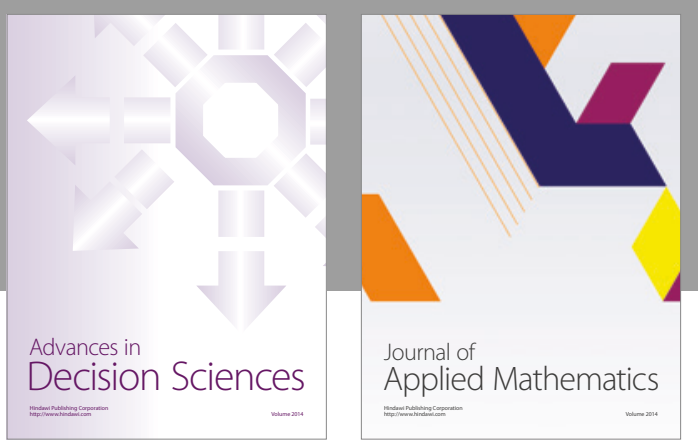

Algebra

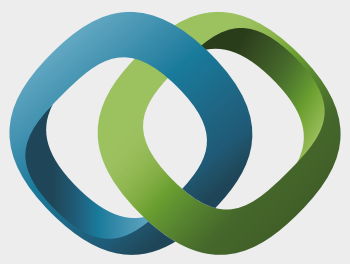

\section{Hindawi}

Submit your manuscripts at

https://www.hindawi.com
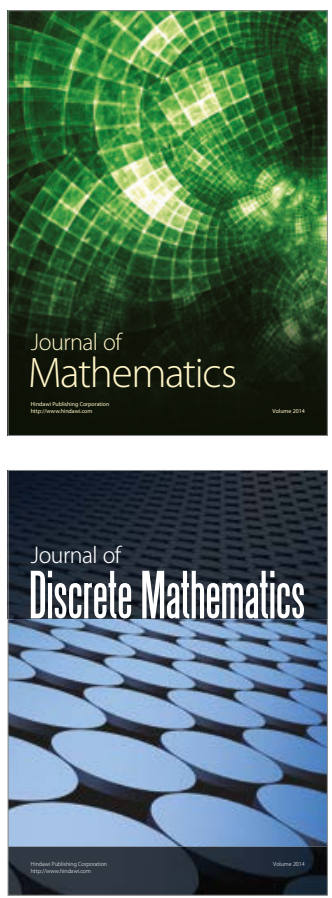

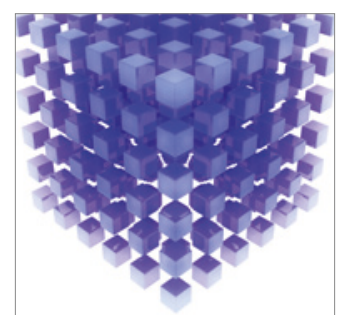

Mathematical Problems in Engineering
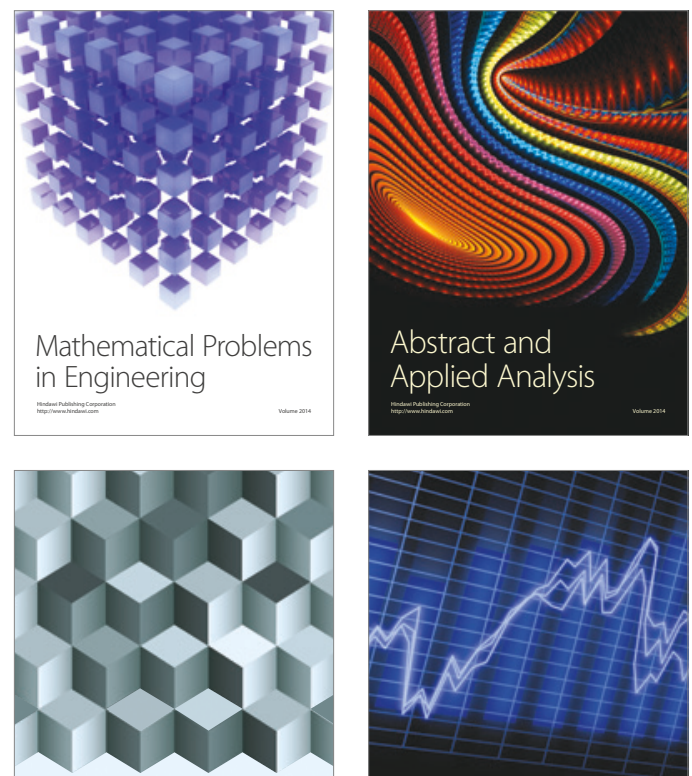

Journal of

Function Spaces

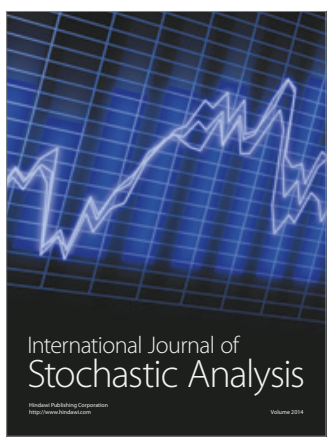

Probability and Statistics
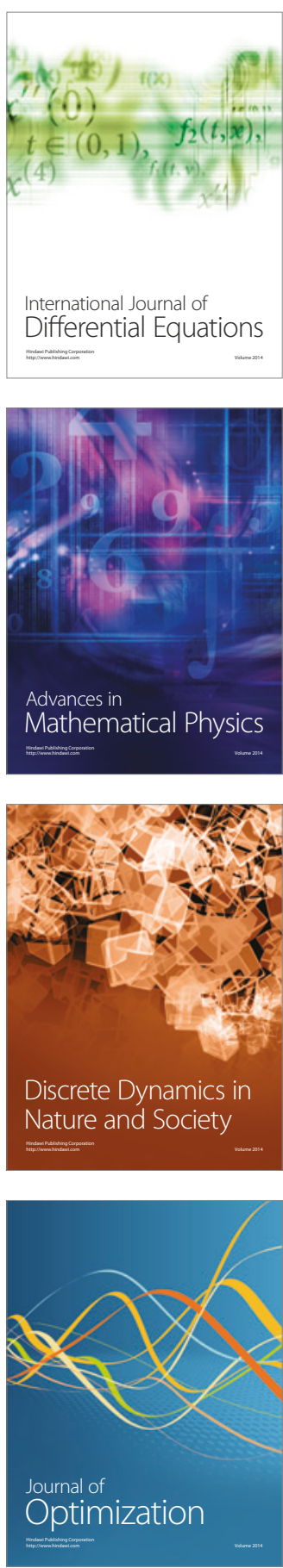